\title{
Brain immune cells undergo cGAS/STING-dependent apoptosis during herpes simplex virus type 1 infection to limit type I IFN production
}

\author{
Line S. Reinert, ${ }^{1}$ Ahmad S. Rashidi, ${ }^{1,2}$ Diana N. Tran, ${ }^{2}$ Georgios Katzilieris-Petras, ${ }^{1}$ Astrid K. Hvidt, ${ }^{1}$ Mette Gohr, ${ }^{1}$ \\ Stefanie Fruhwürth, ${ }^{3}$ Chiranjeevi Bodda, ${ }^{1}$ Martin K. Thomsen, ${ }^{1}$ Mikkel H. Vendelbo, ${ }^{1,4}$ Ahmad R. Khan, ${ }^{5,6}$ Brian Hansen, ${ }^{5}$ \\ Petra Bergström, ${ }^{7}$ Lotta Agholme, ${ }^{7}$ Trine H. Mogensen, ${ }^{1}$ Maria H. Christensen, ${ }^{8}$ Jens R. Nyengaard, ${ }^{9}$ Ganes C. Sen, ${ }^{10}$ \\ Henrik Zetterberg, ${ }^{3,11,12}$ Georges MGM Verjans, ${ }^{2}$ and Søren R. Paludan ${ }^{1,3}$
}

\begin{abstract}
'Department of Biomedicine, Aarhus University, Aarhus, Denmark. ²Department of Viroscience, Erasmus Medical Centre, Rotterdam, Netherlands. ${ }^{3}$ Department of Psychiatry and Neurochemistry, Institute of Neuroscience and Physiology, Sahlgrenska Academy at the University of Cothenburg, Sweden. ${ }^{4}$ Department of Nuclear Medicine \& PET Centre, Aarhus University Hospital, Denmark. ${ }^{5}$ Center of Functionally Integrative Neuroscience, Aarhus University, Aarhus, Denmark. ${ }^{6}$ Centre of Biomedical Research, SCPGI Campus, Lucknow, India. ${ }^{7}$ Institute of Neuroscience and Physiology, Department of Psychiatry and Neurochemistry, Sahlgrenska Academy at the University of Gothenburg, Gothenburg, Germany. ${ }^{8}$ Institute of Innate Immunity, University of Bonn, Bonn, Germany. ${ }^{9}$ Department of Clinical Medicine, University of Aarhus, Aarhus, Denmark. ${ }^{10}$ Department of Immunology, Lerner Research Institute, Cleveland Clinic, Cleveland, Ohio, USA. "Department of Neurodegenerative Disease, UCL Institute of Neurology, Queen Square, London, United Kingdom. ${ }^{12}$ UK Dementia Research Institute at UCL, London, United Kingdom.
\end{abstract}

\begin{abstract}
Protection of the brain from viral infections involves the type I IFN (IFN-I) system, defects in which render humans susceptible to herpes simplex encephalitis (HSE). However, excessive cerebral IFN-I levels lead to pathologies, suggesting the need for tight regulation of responses. Based on data from mouse models, human HSE cases, and primary cell culture systems, we showed that microglia and other immune cells undergo apoptosis in the HSV-1-infected brain through a mechanism dependent on the cyclic GMP-AMP synthase/stimulator of interferon genes (cGAS/STING) pathway, but independent of IFN-I. HSV-1 infection of microglia induced cGAS-dependent apoptosis at high viral doses, whereas lower viral doses led to IFN-I responses. Importantly, inhibition of caspase activity prevented microglial cell death and augmented IFN-I responses. Accordingly, HSV-1-infected organotypic brain slices or mice treated with a caspase inhibitor exhibited lower viral load and an improved infection outcome. Collectively, we identify an activation-induced apoptosis program in brain immune cells that downmodulates local immune responses.
\end{abstract}

\section{Introduction}

Acute viral encephalitis is a devastating disease and a major cause of severe illness and even death (1). Herpes simplex virus type 1 (HSV-1) is a neurotropic human alpha herpesvirus and is the primary cause of viral encephalitis in the Western world (2). The immune system is the key host entity that controls HSV-1 infections in the periphery and in the brain. Animal models combined with mouse genetics have revealed particularly important roles for the type I IFN (IFN-I) system for control of primary infections and $\mathrm{CD} 8^{+} \mathrm{T}$ cells for control of viral latency $(3,4)$. HSV- 1 enters the brain via infection of peripheral sensory neurons and subsequent anterograde axon transport to the CNS (5). The virus replicates in neurons, but tropism for astrocytes, for example, has also been demonstrated $(6,7)$. The brain is an organ that does not tolerate much damage, and tissue repair cannot reestablish homeostasis in the same way as observed in other organs like the liver and skin $(8$,

Authorship note: ASR and DNT contributed equally to this work

Conflict of interest: The authors have declared that no conflict of interest exists.

Copyright: @ 2021, American Society for Clinical Investigation.

Submitted: January 28, 2020; Accepted: September 23, 2020; Published: January 4, 2021.

Reference information: J Clin Invest. 2021;131(1):e136824.

https://doi.org/10.1172/JCl136824.
9). Therefore, in addition to the acute symptoms of viral encephalitis, such as altered mental status, fever, seizures, and neurological deficits, the long-term neurological sequela for survivors are often highly disabling (1). To prevent brain damage in response to infections, the brain abundantly uses cell-preserving and cell-autonomous mechanisms to control virus infections (10-13), and the more disruptive antiviral activities must be tightly controlled.

Genetic analyses of patients with herpes simplex encephalitis (HSE) have demonstrated an essential role for the IFN-I system and TLR3-mediated IFN-inducing pathways in the prevention of HSE (14-18). Three prime IFN-inducing pathways known are TLR3/TIR domain-containing adapter-inducing IFN- $\beta$ (TLR3/ TRIF) (19, 20), retinoic acid-inducible gene I/mitochondrial antiviral signaling protein (RIG-I/MAVS) (21, 22), and cyclic GMP-AMP synthase/stimulator of interferon genes (cGAS/ STING) $(23,24)$. TLR3 senses dsRNA in endosomes, RIG-I senses dsRNA in the cytoplasm, and cGAS senses dsDNA in the cytoplasm. We previously reported that cGAS and STING are highly expressed in microglia, which utilize this pathway to produce the bulk of IFN-I in the HSV-1-infected mouse brain (25). Consequently, the cGAS/STING pathway is essential for control of HSV-1 infection in mouse models for HSV-1 brain infection (2527). In addition to the antiviral function of IFN-I, however, this 
family of cytokines also contributes to immunopathology, with the brain being a particularly sensitive organ to long-term effects of IFN-I activity (28). This is illustrated by the autoinflammatory disease Aicardi-Goutieres syndrome, which is an encephalopathy driven by IFN-inducing signaling pathways sharing some features with congenital viral infections, including intracranial calcifications (29). Therefore, regulatory mechanisms limiting IFN-I responses may protect the brain from irreversible damage.

Microglia are resident immune cells of the brain (30). This cell type is important for host defense and homeostasis in the brain (31-33), as well as for proper development of several core activities of the brain, including learning-dependent synapse formation (34). However, microglia are also involved in numerous pathological processes. For instance, in mouse models of sterile encephalitis and tauopathy, depletion of microglia attenuates pathology $(35,36)$. The immunological activities of microglia include production of cytokines and ROS, phagocytosis, and recruitment of peripheral immune cells. All these processes can have both beneficial and deleterious effects (30). Currently, there is a limited knowledge on how microglia and other immune cells balance immune responses in the brain to achieve the favorable effects of a given activity and minimize pathology.

HSV-1 has a double-stranded linear DNA genome, and in recent years it has emerged that DNA-sensing receptors not only induce IFN-I expression, but also other immunological activities, including expression of inflammatory cytokines, autophagy, and different types of programmed cell death (PCD) (37). This includes apoptosis, autophagic cell death, lysosomal cell death, necroptosis, and pyroptosis (38-43). Interestingly, the cGAS/ STING pathway can induce all these modalities of PCD (37), and for STING-dependent apoptosis there is evidence for this occurring either directly through interaction of IFN-regulatory factor 3 (IRF3) and BCL2-associated x, apoptosis regulator (Bax) (38) or indirectly through IRF3-dependent transcription of proapoptotic genes, including p53 upregulated modulator of apoptosis (PUMA) (39). However, it remains unresolved what determines the type of PCD that is executed downstream of STING in a given physiological context and what the physiological role is.

In this work, we determined the type of PCD in HSV-1infected brains of human HSE cases and an experimental mouse model, and applied the mouse model to detail its impact on the local immune response. We report that HSV-1 induced cGAS/ STING-dependent apoptosis in microglia and other immune cells at a high viral load, whereas a low-to-medium viral load favored IFN-I expression. Death of brain immune cells downmodulated IFN-I-driven antiviral responses, while potentially limiting their long-term pathological effects.

\section{Results}

$H S V$-1 infection induces cGAS-dependent apoptosis in the murine brain. We have previously reported that $\mathrm{CGas}^{-/}$and Sting ${ }^{-/-}$mice have impaired production of IFN-I and elevated viral load in the brain during HSV-1 infection and are highly susceptible to disease (25). We reproduced these findings (Supplemental Figure 1A; supplemental material available online with this article; https://doi.org/10.1172/ JCI136824DS1), but also observed that there was increased edema in the brain lesions in the $c \mathrm{Gas}^{-/-}$mice (Supplemental Figure 1, B and
C), potentially suggesting elevated inflammation. When examining whole brainstems for IFN-I responses, we found that cGAS was essential for evoking this expression profile (Supplemental Figure 1D), as we have shown previously (25). However, use of laser-capture microdissection to distinguish between brainstem regions with different degrees of infection revealed that mRNA levels of Ifn $b$ and several IFN-stimulated gene (ISG) responses tended to be lower within the highly infected brain areas (Figure 1A, red box). This suggests that alternative immunological activities were induced in the highly infected areas. Interestingly, in these brain areas in WT mice, we detected a high number of TUNEL ${ }^{\text {pos }}$ and propidium iodide-positive ( $\left.\mathrm{PI}^{\mathrm{pos}}\right)$ cells, observed in many types of $\mathrm{PCD}$, including apoptosis, necroptosis, and pyroptosis (44) (Figure 1B and Supplemental Figure 1, E and F). This was not found in $c \mathrm{Gas}^{-/-}$mice. Based on these observations, we hypothesized that a regulatory process involving cGAS and PCD is activated in the highly infected brain regions.

To determine the role of PCD in HSV-1 infection, we first examined which PCD type was induced in the HSV-1-infected brain. Brainstem lysates from infected mice or cultured organotypic brain slices infected with HSV-1 were analyzed for markers of apoptosis (cleaved caspase-3 [CC3]), pyroptosis (cleaved caspase-1 [CC1] and cleaved gasdermin D [CGSDMD]), and necroptosis (phosphorylated mixed-lineage kinase domain-like protein [P-MLKL] and phosphorylated receptor-interacting serine/threonine-protein kinase 3 [P-RIPK3]) (37), by both Western blotting and IHC (Figure 2 and Supplemental Figure 2). By treating organotypic brain slices with agents that induced specific PCD types, including LPS + nigericin (pyroptosis), TNF- $\alpha+$ Z-VAD (necroptosis), and raptinal (apoptosis), we readily detected pyroptosis, necroptosis, and apoptosis, respectively (Supplemental Figure 2, A-D). However, in highly HSV-1-infected areas of both the brainstem of infected mice and organotypic brain slices, we could not detect significant differences in expression of the pyroptosis markers CC1, IL1-b, and CGSDMD (Figure 2, A-D; Supplemental Figure 1G; and Supplemental Figure 2E) and necroptosis markers $\mathrm{P}-\mathrm{MLKL}$ and P-RIPK3 in WT compared with $\mathrm{cGas}^{-/-}$mice (Figure 2, E-G, and Supplemental Figure 2F). Interestingly, HSV-1 infection led to increased total levels of $\mathrm{C} 1$ and MLKL, suggesting that the infection primes the brain for necrotic forms of PCD (Figure 2, C and E, and Supplemental Figure 2G), but C1 and MLKL did not seem to be activated. In sharp contrast, examination of infected mouse brains for the apoptosis marker CC3 revealed a clear cGAS-dependent induction of this response in the infected brain (Figure 2, H and I). This was also observed by IHC and Western blotting of HSV-1-infected organotypic brain slices (Figure 2J and Supplemental Figure $1 \mathrm{H}$ ) and by flow cytometry on brainstem cells from infected mice (Figure 2K). Collectively, these data demonstrated that HSV-1 infection in the mouse brain induced apoptosis in the area of infection in a cGAS-dependent manner.

HSV-1 infection-induced apoptosis in the brain is independent of IFN-I. Similar to $\mathrm{CGas}^{-/-}$mice, Sting ${ }^{-/-}$mice also exhibited impaired induction of caspase-3 cleavage upon HSV-1 infection, demonstrating that virus-induced apoptosis occurred through the cGAS/ STING pathway (Supplemental Figure 3, A and B). To further characterize cGAS/STING-dependent apoptosis in vivo, we first examined the sequence of events in detail. Although no CC3 was observed in the brainstem on day 3 after infection (Supplemental 

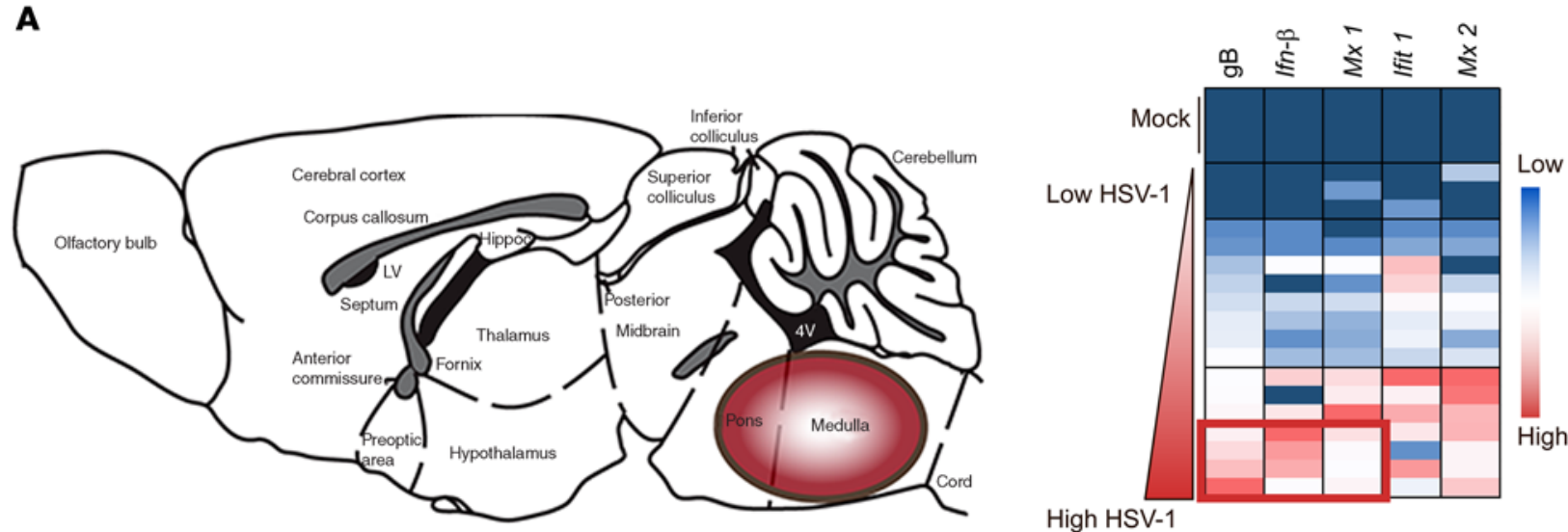

High HSV-1

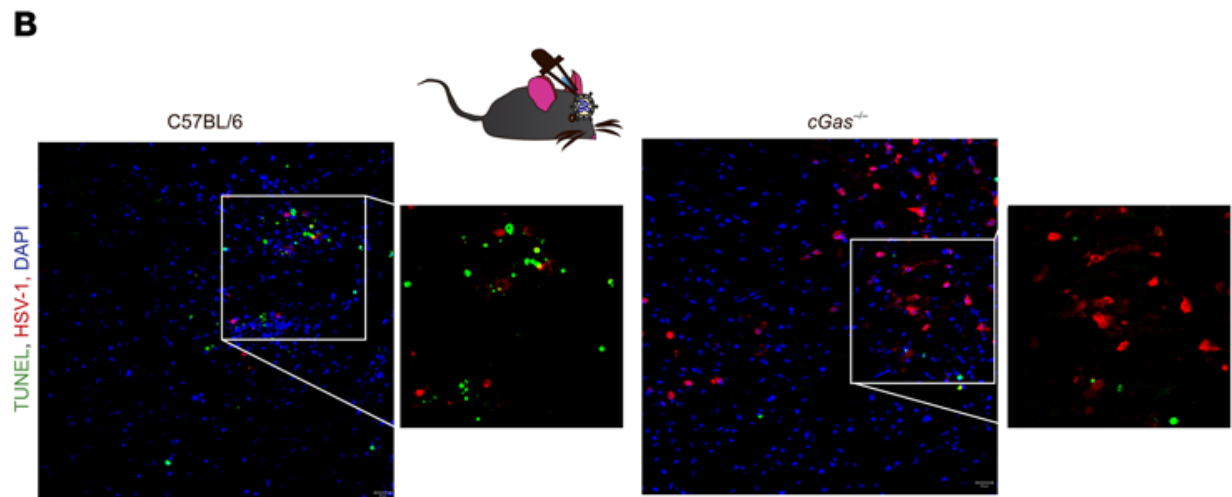

Figure 1. HSV-1 induces cGAS-dependent cell death in the brain microenvironment. Brainstems were isolated at 5 days after HSV-1 infection via the corneal route $\left(2 \times 10^{6} \mathrm{PFU} /\right.$ cornea). (A) IFN/ISG profiles from samples isolated by laser-capture microdissection (LCM). Relative transcript levels of the indicated genes in areas of the brainstem from mock- or HSV-1-infected C57BI/6 mice. The red box indicates degree of infection in the infected brainstem areas subjected to LCM based on IHC staining of the sequential sections. Values are normalized to $\beta$-actin and each row represents 1 LCM preparation ( $n=4-8$ mice per group). Blue and red color indicate low and high expression, respectively. (B) IHC images of brainstems from HSV-1-infected mice stained for TUNEL (green) and HSV-1 VP5 (red). Scale bar: $10 \mu \mathrm{m}$. Areas marked by squares are magnified in the images to the right of the large images. Animals per group: $n=6-7$.

Figure 3C), detectable levels of apoptosis started to appear at day 4 after infection, and further increased on day 5 after infection (Figure 3, A-C). This correlated with the HSV-1 infection level of the brain (Figure 3D). Next, we explored the dependency on the canonical IFN-I response, which is induced in a cGAS/STING-dependent manner in the HSV-1-infected murine brain (25). Interestingly, although Ifnar $^{-/}$mice (deficient in IFN- $\alpha / \beta$ receptor [IFNAR]) exhibited significantly elevated susceptibility to infection and ablated ISG induction (Supplemental Figure 3, D-I), the apoptotic response was not significantly reduced in the brainstem of HSV-1-infected Ifnar ${ }^{--}$mice (Figure 3, E-H). Because IRF3 is downstream in the STING pathway (45), we wanted to examine whether IRF3 and its transcriptional activity were important for the virus-induced apoptosis response in the HSV-1-infected brain. For this purpose, we compared WT, $\operatorname{Irf3^{-/-}}$, and $\operatorname{Irf} 3^{s 1 / s 1}$ mice; the latter mouse strain cannot activate IRF3 transcription but can induce apoptosis through the IRF3-mediated pathway of apoptosis (RIPA) $(46,47)$. Both $\operatorname{Irf} 3^{-/-}$and $\operatorname{Irf} 3^{s 1 / s 1}$ mice were more susceptible to HSV-1 brain infection than WT mice (Supplemental Figure $3, \mathrm{~J}-\mathrm{L}$ ) and expressed lower levels of Ifnb (Supplemental Figure $3 \mathrm{M})$. Interestingly, we also observed significantly less activation of the apoptotic response in both of these mouse strains (Figure
3, I-L). These results suggest that HSV-1 induces apoptosis in the brain through the cGAS/STING pathway independent of IFN-I but dependent on IRF3 transcription.

Microglia and other immune cells are the major cell types undergoing apoptosis in the HSV-1-infected brain. To identify the cell types that undergo cGAS-dependent cell death in the HSV-1infected brain, we stained brainstem tissue sections from WT and $c \mathrm{Gas}^{-/-}$mice obtained at day 5 after infection with antiCC3; DAPI; and markers of the major brain cell types, including neurons (NeuN), astrocytes (GFAP), microglia (Iba1), and leukocytes (CD45) (Figure 4A). We observed no colocalization of CC3 and NeuN (Figure 4B) and only very little between CC3 and GFAP (Figure 4C), although these 2 cell types were abundantly HSV-1 infected (25). By contrast, we noted prominent CC3 staining in microglia (Figure 4D) and even more in CD45 pos cells representing both microglia and brain-resident/infiltrating immune cells (Figure 4E). When brain tissue from day 4 after infection was analyzed, we observed a higher percentage of $\mathrm{CC} 3^{\text {pos }}$ cells, again predominantly microglia (Figure $4 \mathrm{~F}$ ). About $70 \%$ of $\mathrm{CC}^{\text {pos }}$ cells detected by IHC in HSV-1-infected mouse brains appeared to be apoptotic immune cells (CD45 $5^{\text {pos }}$ ), including microglia (Table 1). These data indicate that microglia and 
A
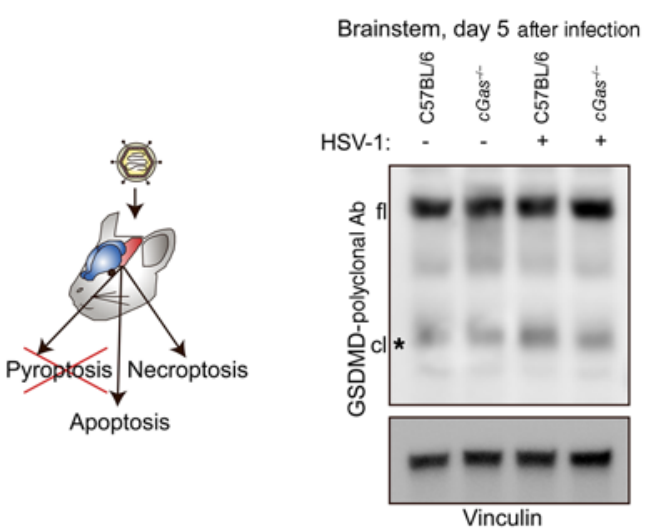

B

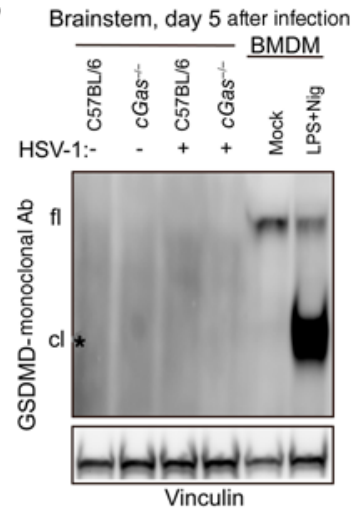

E
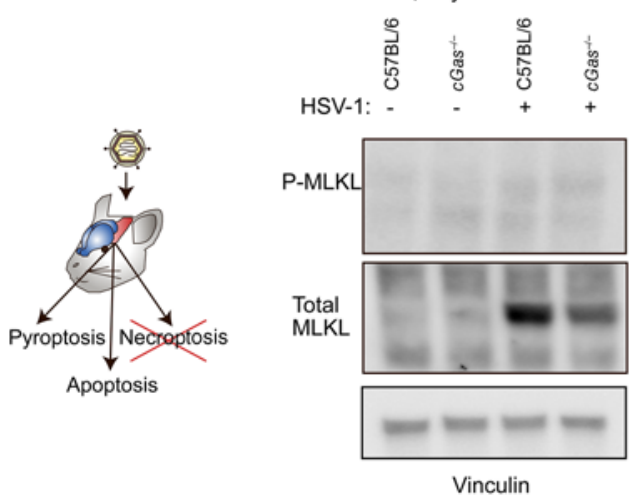

Brainstem, day 5 after infection
C Brainstem, day 5 after infection

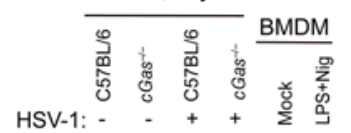

C1-
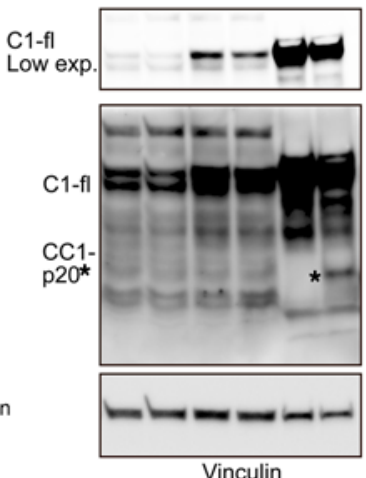

$\mathbf{F}$

Brainstem, day 5 after infection

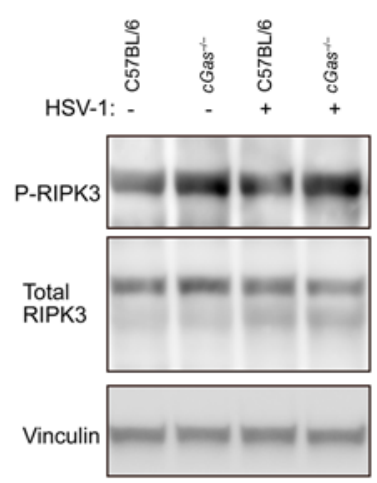

I

Brainstem, day 5 after infection

$$
\boldsymbol{m}_{\mathrm{CGas}^{+-}}^{\mathrm{C} 57 \mathrm{BL} / 6}
$$

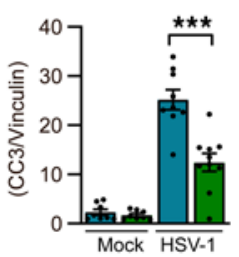

G

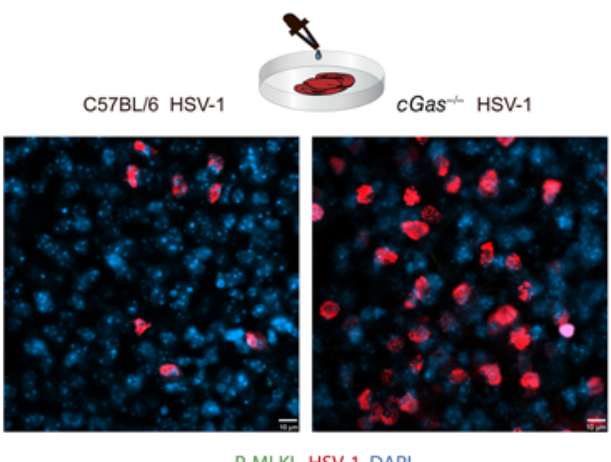

P-MLKL, HSV-1, DAPI

J
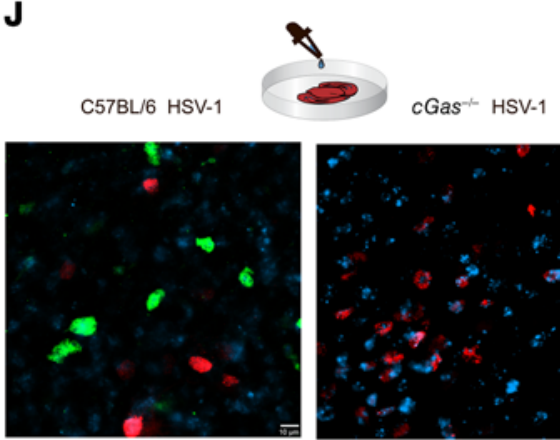

CC3, HSV-1, DAPI

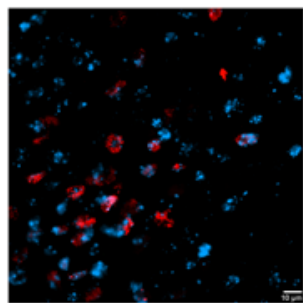

H

Brainstem, day 5 after infection

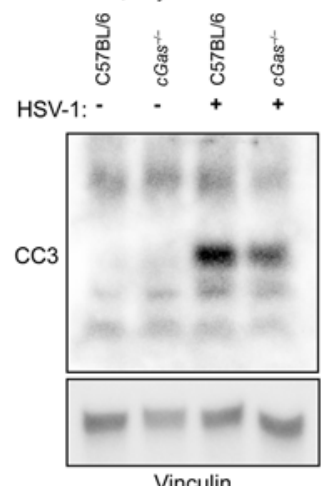

$\mathbf{K}$

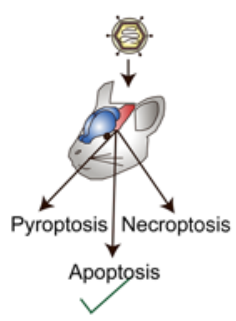

Brainstem, day 5 after infection

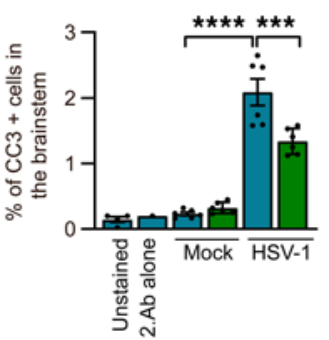


Figure 2. The cGAS-dependent cell death in the HSV-1-infected brain is primarily apoptosis. (A-C, E, F, and $\mathbf{H}$ ) Immunoblots for the indicated PCD markers in the brainstem from HSV-1-infected mice at day 5 after infection; vinculin was used as a loading control. (A and $\mathbf{B}$ ) Both full-length gasdermin $\mathrm{D}$ (GSDMD) (fl) and the cleaved (CGSDMD) ( $\mathrm{Cl}^{*}$ ) product in the brainstem were detected with a polyclonal antibody (A), while the monoclonal antibody used (B) detects predominately the CGSDMD $\left(\mathrm{Cl}^{*}\right)$ product. The positive control used was bone marrow-derived macrophages (BMDM) treated in vitro with LPS $(1 \mu \mathrm{g} / \mathrm{mL})$ for 4 hours followed by an additional treatment with nigericin for 1 hour $(10 \mu \mathrm{M})$. (C) Immunoblotting for caspase-1 showing the full-length caspase- $1(\mathrm{C} 1-\mathrm{fl})$ and the cleaved caspase- 1 (P20)*. BMDM cells were treated as in B. (D, G, and J) Organotypic brain slices from WT and $\mathrm{CGas}^{-/-}$mice were cultured and infected with $1 \times 10^{4} \mathrm{PFU}$ of HSV-1 for 20 hours, fixed and stained for HSV-1 (DP5) (red), GSDMD (monoclonal) antibody, P-MLKL, or cleaved caspase-3 (CC3), as indicated (all shown in green). Scale bar: $10 \mu \mathrm{m}$. (E and F) Immunoblots for the necroptosis markers P-MLKL and P-RIPK3 together with vinculin, total MLKL, and total RIPK3. ( $H$ and I) Immunoblotting for and quantification of CC3 bands normalized to vinculin bands presented as mean \pm SEM per group $(n=8-10), P$ values were calculated by Wilcoxon rank-sum test. ${ }^{* *} 0.0001<P<0.001$. (K) Levels of CC3 in all cells present in the brainstem were analyzed by flow cytometry and presented as mean \pm SEM. $n=1-6$ per group, $P$ values were calculated by 1-way ANOVA with Tukey's multiplecomparison test. ${ }^{* *} 0.0001<P<0.001$; ${ }^{* * *} P<0.0001$. All results presented in this figure are representative for at least 3 independent experiments.

other immune cells in highly infected areas of HSV-1-infected mouse brains underwent cGAS-dependent apoptosis.

Apoptosis of immune cells is a major type of PCD in brain tissue of HSE patients. To further validate the HSE mouse model, we determined whether similar PCD pathways are involved in HSE patients by performing detailed IHC on HSV-1-infected brain tissue of 5 fatal HSE cases. When we stained for TUNEL and HSV-1, we observed extensive cell death in the highly infected areas of HSE cases, but not in areas of low infection from the same patients (Figure 5, A and B). This was also observed when we stained for CC3 (Figure 5, C and D). By contrast, GSDMD and P-MLKL expression were undetectable by IHC in the same brain regions of the HSE cases (Figure 5E), whereas both PCD markers were readily detected in sections from a patient dying from brain trauma unrelated to infection (Figure 5F).

To investigate whether microglia and other immune cells are major cell types undergoing cell death in the HSE brain, we determined coexpression of TUNEL and various cell-type markers. Analogous to the HSE mouse model, we observed prominent death of Iba- $1^{\text {pos }}$ and CD $45^{\text {pos }}$ cells in highly infected areas of HSE brains (Figure 5, G and H, and Supplemental Figure 4, A and B). In addition, we generally observed minimal cell death among astrocytes, similar to what was found in mouse brains, although in 2 of 5 HSE cases, relatively high frequencies of TUNEL-GFAP double-positive cells were detected (Supplemental Figure 4, C and D). We observed significant interpatient variation in the level of infection (Supplemental Figure 4E). While the majority of HSV$1^{\text {pos }}$ cells were also CC 3 pos (Supplemental Figure $4 \mathrm{~F}$ ), we additionally observed higher levels of TUNEL ${ }^{\text {pos }}$ debris than $\mathrm{CC}^{\text {pos }}{ }^{\text {pells }}$ (Supplemental Figure 4G for human and Supplemental Figure 4H for mice), potentially representing phagocytosed cell debris or late apoptotic cells with lost expression of cell-specific markers. The combined data indicate that in both the experimental HSE mouse model and human HSE cases, HSV-1 infection of the brain led to extensive apoptotic cell death in microglia and other immune cells, particularly in the highly infected areas of the brain.

Microglia undergo apoptosis through the mitochondrial pathway in response to high HSV-1 doses. With the observation that microglia and other immune cells undergo cGAS-dependent apoptosis in the HSV-1-infected brain, we explored whether this is an intrinsic property of these cells. Therefore, murine microglia from WT and $c \mathrm{Gas}^{-/}$mice were isolated, cultured, and HSV-1 infected. As was seen in vivo, HSV-1 infection induced cGAS-dependent apoptosis (Figure 6, A and B) without disintegration of the plasma membrane (Figure 6C). While $c \mathrm{Gas}^{-/}$microglia had slightly higher transcription of the HSV-1 late gene gB than WT microglia (Figure 6D), virus infection did not induce Ifnb expression (Figure 6E). Interestingly, we also observed that HSV-1-infected microglia induced transcription of the proapoptotic gene Puma in a cGAS-dependent manner (Figure 6F). PUMA has previously been proposed to be involved in STING-dependent apoptosis (39). In order to evaluate whether human microglia behave in a similar manner, we generated iPSC-derived microglia and infected them with HSV-1 or treated them with the STING agonist 2' $3^{\prime}$ cGAMP. Indeed, HSV-1 infection induced CC3 activity in a dose-dependent manner, as did activation of STING signaling (Figure 6G).

Because of the observed trend toward viral load correlating inversely with Ifnb mRNA levels in highly infected areas (Figure $1 \mathrm{~A})$, we questioned whether the 2 cGAS-dependent virus-induced events, apoptosis and Ifnb transcription, exert different HSV-1 dose dependency. Interestingly, while HSV-1-induced CC3 activity increased with viral dose increment (Figure 6H), Ifnb expression peaked at low doses of infection and decreased with higher MOI (Figure 6I). In order to examine whether the observed apoptosis was triggered by the intrinsic or the extrinsic pathway, we first tested for release of cytochrome $c$ into the cytoplasm (48). For this purpose, we used the murine microglia-like cell line BV2, given the requirement for a larger number of cells that could easily be obtained from mice. In BV2 cells, apoptosis was induced by a STING agonist and dsDNA (Supplemental Figure 5A), and HSV-1induced CC3 activity and Ifnb expression showed the same dose dependency as in primary mouse microglia (Supplemental Figure 5, B and C). Upon HSV-1 infection of BV2 cells and primary mouse microglia, cytochrome $\mathrm{c}$ was relocated from the mitochondria to the cytoplasm (Figure 6, J and K, and Supplemental Figure 5E). By contrast, cytokines induced by HSV-1 infection of BV2 cells did not induce CC3 activity, nor did IFN- $\beta$ augment apoptosis triggered by STING agonist treatment (Supplemental Figure 5D). Collectively, these data indicate that low-intermediate stimulation of the cGAS/ STING pathway in brain immune cells induced IFN-I expression, whereas a high load of STING agonists favored signaling to apoptosis through the intrinsic pathway (Figure 6L).

HSV-1 infection or HSV-1-infected brain cells induce microglia apoptosis, thereby reducing IFN- $\beta$ expression. Based on the observation that there was inverse correlation between the IFN-I and apoptosis responses, we wanted to examine the potential functional interaction between both responses in cells undergoing apoptosis in vivo. For this purpose, $W T$ and $c \mathrm{Gas}^{-/}$microglia or splenocytes $\left(-90 \% \mathrm{CD} 45^{\text {pos }}\right.$ cells) were HSV-1 infected in the presence or absence of a pan-caspase inhibitor zVAD. This treatment prevented virus-induced activation of caspase-3/7 and cell death both in 
A
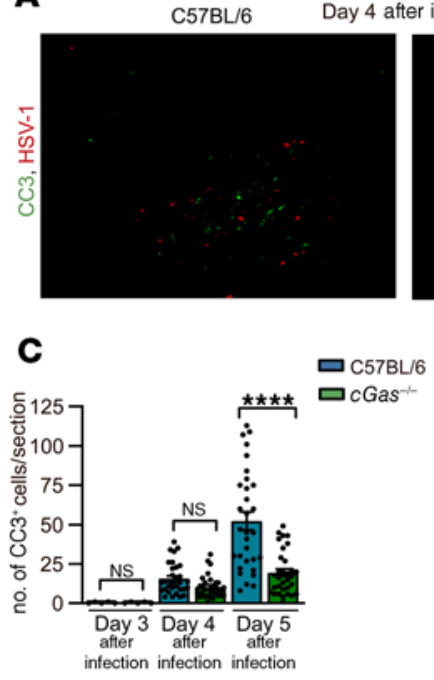

$\mathbf{F}$

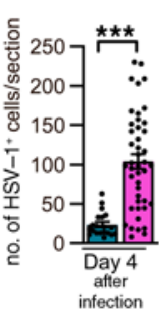

I

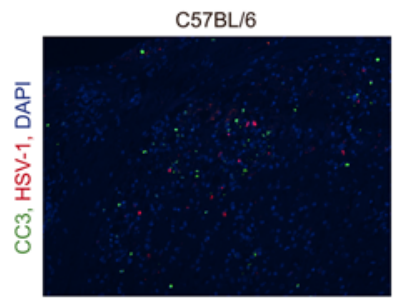

G

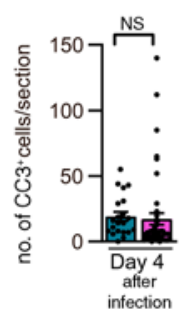

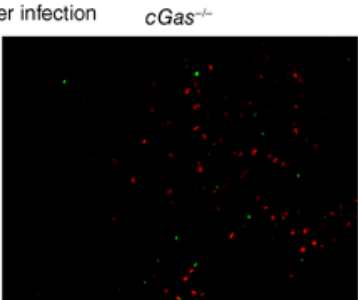

D

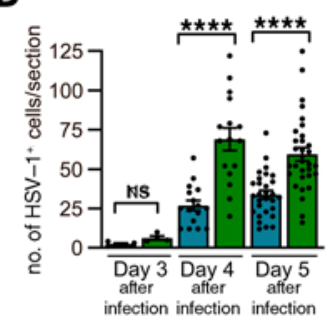

H

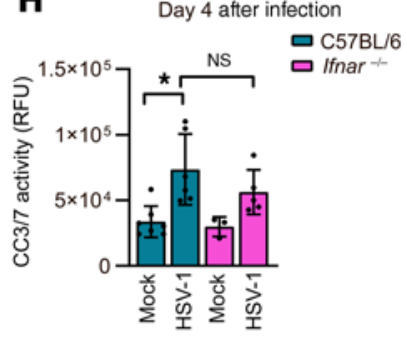

Day 5 after infection IIf3

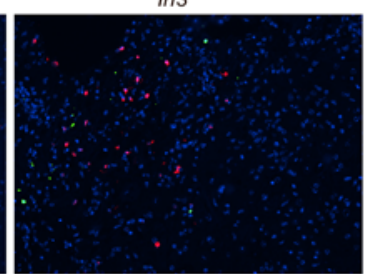

B
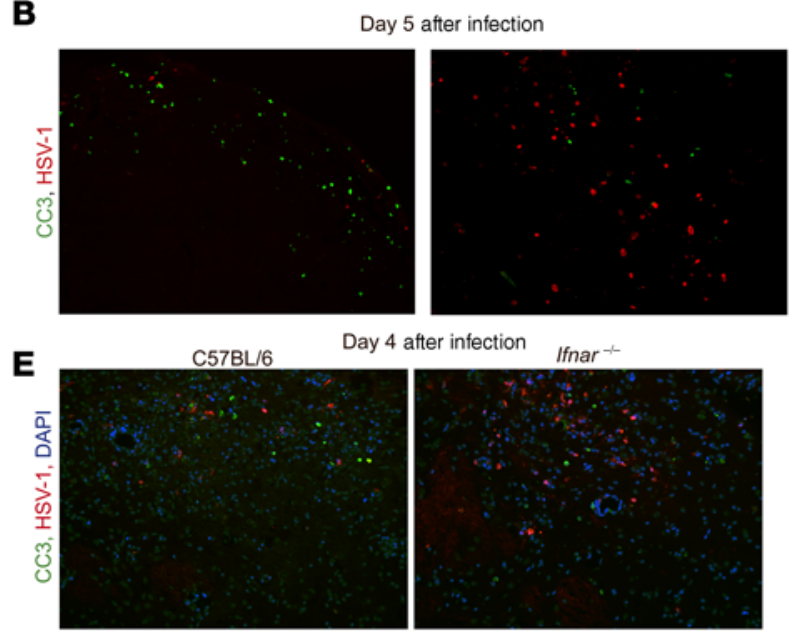

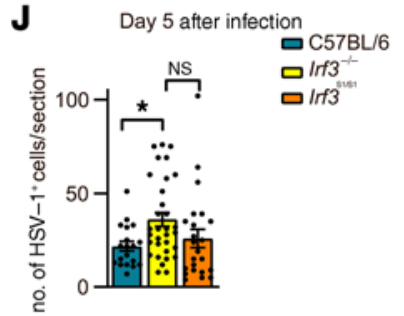

$\mathbf{K}$

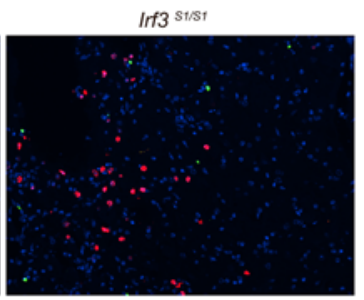

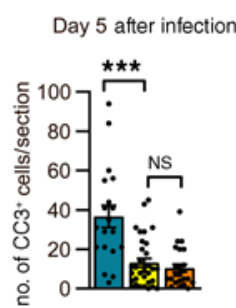

$\mathbf{L}$

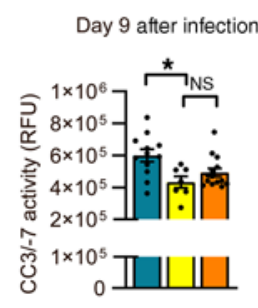

Figure 3. HSV-1-induced apoptosis in the brain is independent of IFNAR but dependent on IRF3. (A and B) WT and cGas ${ }^{-1-}$ mice were infected with HSV-1 $\left(2 \times 10^{6} \mathrm{PFU} /\right.$ cornea) and the brainstems dissected at either (A) day 4 or (B) day 5 after infection. Tissue sections were stained for HSV-1 (VP-5, red) and cleaved caspase-3 (СС3) (green). Representative images are shown in $\mathbf{A}$ and $\mathbf{B}$ and quantification of CC ${ }^{\text {pos }}$ cells and HSV-1-infected cells per section presented as mean \pm SEM (C and $\mathbf{D}), n=5-32$ images per group, and 5-8 animals per group. (E-C) CC3 and virus levels in the brainstem of WT and Ifnar ${ }^{-/-}$mice detected as in A, C, and $\mathbf{D}$ at day 4 after infection, $n=20-44$ images per group, and 4-5 animals per group. (I-K) CC3 and virus levels in the brainstem of WT and $I r f 3^{-/-}$and $/ r f 3^{51 / 51}$ mice detected as in $\mathbf{B}-\mathbf{D}$ at day 5 after infection. $n=19-32$ images per group, and $4-8$ animals per group. (H and $\mathbf{L}$ ) Brainstem homogenates from HSV-1-infected mice were analyzed for CC3 activity and data presented as mean \pm SEM, $n=3-15$ per group. $P$ values were calculated using 2-way ANOVA with Bonferroni's post hoc test (C and $\mathbf{D})$, 1-way ANOVA with Kruskal-Wallis multiple-comparison test ( $\mathbf{H}$ and $\mathbf{L})$, and Wilcoxon rank-sum test $(\mathbf{F}$ and G). For $\mathbf{D}, \mathbf{F}$, and J, only sections with clear HSV-1pos cells were counted. All results presented in this figure are representative of at least 3 independent experiments; original magnification, $\times 20 . P>0.05$ (NS); ${ }^{*} 0.01<P<0.05 ;{ }^{* *} 0.001<P<0.01 ;{ }^{* *} 0.0001<P<0.001 ;{ }^{* * *} P<0.0001$.

microglia and CD45 $5^{\text {pos }}$ cells in a dose-dependent manner in WT but not CGAS-/- mice (Supplemental Figure 6, A-C). Interestingly, it also led to elevated HSV-1-induced expression of Ifnb and Cxcl1O in WT mice (Figure 7, A and B, and Supplemental Figure 6D) and further reduction of the already modest viral replication observed in microglia (Supplemental Figure 6E). This was independent of cGAS cleavage, as recently suggested in immortalized bone marrowderived macrophages and HeLa cells (49) (Supplemental Figure $6 \mathrm{~F})$. A similar inverse relationship between apoptosis and IFN-I is also seen in CD45 ${ }^{\text {pos }}$ splenocytes at high MOI compared with 5 MOI (Supplemental Figure 6D). To test whether the observed cross-talk between apoptosis and IFN-I expression also occurred in brain slice cultures, we first infected mixed neuron-astrocyte cultures with HSV-1 and subsequently inactivated the virus by UV treatment followed by addition of uninfected microglia. In this system, microglia phagocytose infected CNS cells, as evidenced by viral antigen in vesicle-like structures in the cytoplasm of Iba1 ${ }^{\text {pos }}$ cells and depletion of nonmicroglial cells from the culture (Figure 7C and Supplemental Figure 6G). Importantly, IHC of the brainstem from HSV-1-infected mice showed viral antigen in the extranuclear region of Iba1 ${ }^{\text {pos }}$ cells (Figure 7D). The data suggest that microglia phagocytose virusinfected cells both in vitro and in vivo. The response of microglia 
A

In vivo, cornea infection

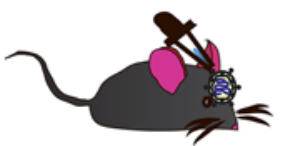

Day 4 or 5 after infection
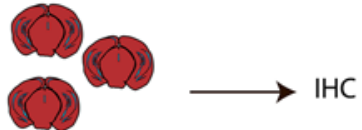

$10 \mu \mathrm{m}$ brainstem sections

\section{B}

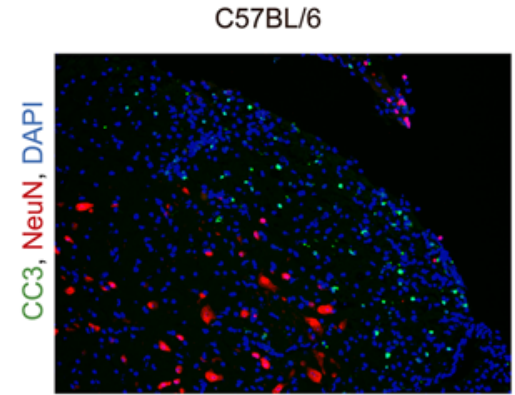

Day 5 after infection
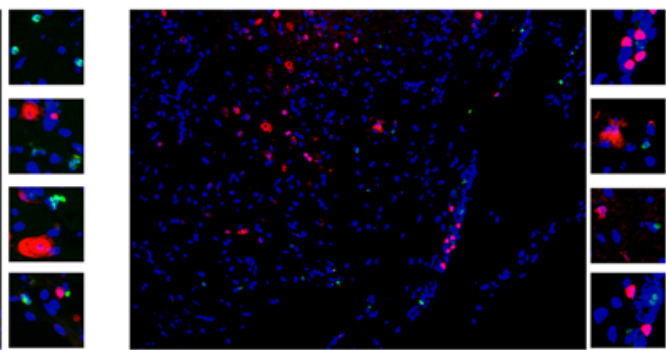

C

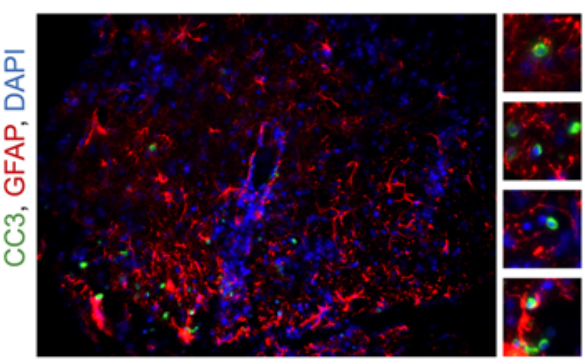

D

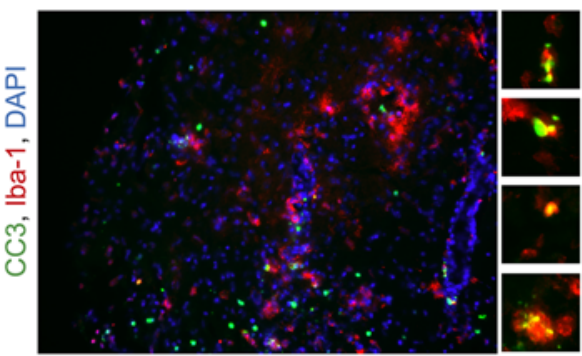

E
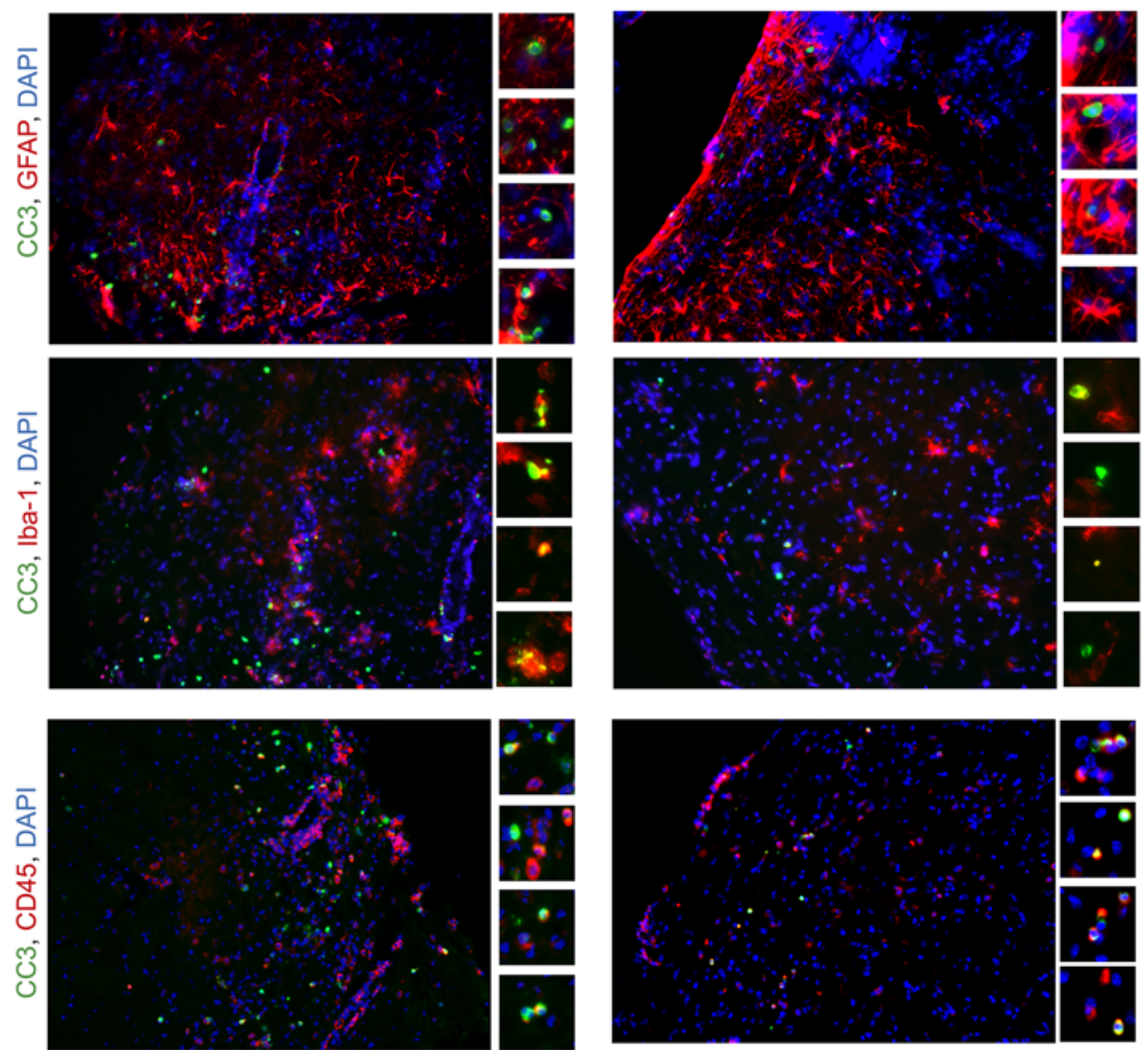

$\mathbf{F}$

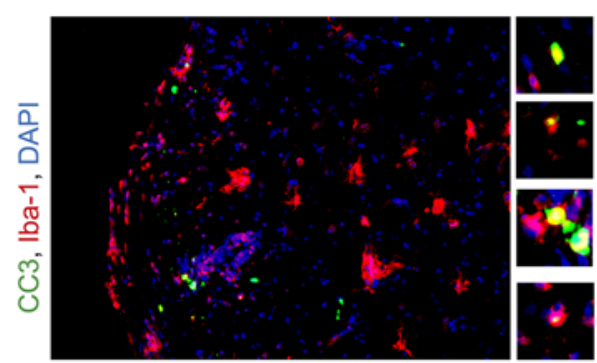

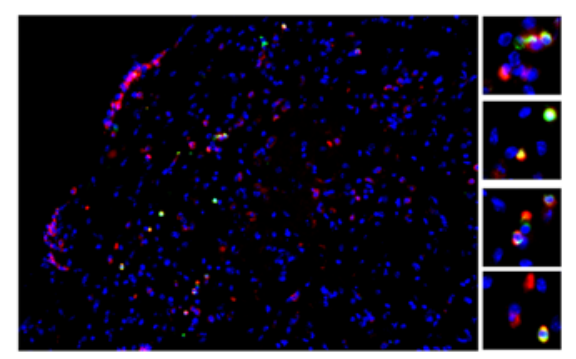

Day 4 after infection

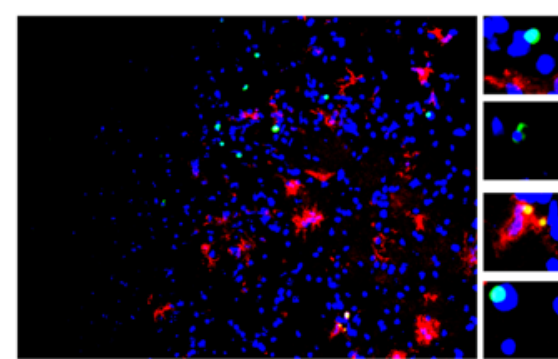

Figure 4. Microglia and other immune cells undergo apoptosis in vivo. (A) Tissue sections from the brainstem of WT and cCas $^{-1-}$ mice infected with HSV-1 (2 $\times$ $10^{6} \mathrm{PFU} /$ cornea) for (B-E) 5 or (F) 4 days were stained with DAPI (blue), antibodies against cleaved caspase-3 (CС3, green) and the following cell type-specific markers (all in red): (B) NeuN (neurons), (C) GFAP (astrocyte), (D and F) Iba1 (microglia), or (E) CD45 (microglia and other immune cells). Cells are magnified in the images to the right of the large images. All results presented in this figure are representative of at least 3 independent experiments; original magnification, $\times 20,(n=12-16$ sections from 6-8 mice per group).

upon coculture with infected brain cells demonstrated elevated CC3 activity and Ifnb expression (Figure 7, $\mathrm{E}$ and $\mathrm{F}$ ), which occurred in a cGAS-dependent manner (Figure $7 G$ and Supplemental Figure $6 \mathrm{H})$. Inhibition of caspase activity upon addition of mixed UV-irradiated HSV-1-infected brain cells to naive microglia further increased Ifnb expression (Figure $7 \mathrm{H}$ ). These data suggest that HSV-1 infection of microglia or phagocytosis of infected cells induced their apoptosis, which terminated the microglial IFN-I response (Figure 7I).

Blockage of caspase activity in the brain improves HSV-1 clearance and outcome of infection. Finally, we wanted to test the effect of caspase inhibition on outcome of HSV-1 infection in the brain. We used a caspase- 3 inhibitor, Q-VD-Oph, which has been demonstrated to have limited toxic effects and to efficiently cross the blood-brain barrier in vivo (50). We first treated organotypic brain slices with the caspase inhibitor and determined its effect on HSV-1 replication. Caspase inhibition led to significant inhibition of HSV-1 replication (Figure 8A), while simultaneously increasing IFN-I response in the brain slices (Figure 8B and Supplemental Figure 7A). Next, we infected mice with HSV-1 via the corneal ocular route with high doses of HSV-1 in order to see severe symptoms and then treated the mice with Q-VD-Oph (Figure 8, C and D, and Supplemental 
Table 1. Mainly microglia and immune cells undergo apoptosis in vivo

\begin{tabular}{|c|c|}
\hline \multicolumn{2}{|c|}{ Average percentage of $\mathrm{CC} 3^{+}$cells expressing cell type-specific markers: } \\
\hline Neurons (NeuN) & $0 \%$ \\
\hline Astrocytes (GFAP) & $1.64 \%[ \pm 2.57 \%]$ \\
\hline Microglia cells (Iba-1) (day 5 after infection) & $13 \%[ \pm 5 \%]$ \\
\hline Microglia cells (Iba-1) (day 4 after infection) & $24 \%[ \pm 10 \%]$ \\
\hline Microglia and immune cells (CD45) & $71 \%[ \pm 11 \%]$ \\
\hline
\end{tabular}

Quantification of images of the HSV-1-infected C57BI/6 brainstem sections such as the representative images shown in Figure 4, B-F. The numbers are the mean percentage of CC $3^{\text {pos }}$ cells containing the respective cell marker; data are shown as mean \pm SEM ( $n=12-16$ sections from $6-8$ mice per group).

Figure 7B). Inhibition of caspase activity in vivo led to significant improvement of the clinical phenotype, illustrated by limiting head swelling and weight loss due to HSV-1 infection (Figure 8, C and D). Importantly, this was associated with decreased viral load in the brainstem of Q-VD-Oph-treated WT but not $c \mathrm{Gas}^{-/}$mice (Figure $8 \mathrm{E}$ ). To examine in more detail the intracerebral virus-induced If $n-\beta$ response, we inoculated mice with GFP-HSV-1, a recombinant HSV-1 strain expressing GFP in productively infected cells together with $\mathrm{zVAD}$ via the intracranial route to deliver the virus directly and locally into the brain. Upon dissection of the brain, GFP-expressing regions were biopsied and analyzed for virus and host markers (Figure 8, F and G). The GFP signal correlated positively with HSV-1 gB transcription, demonstrating that the GFP signal is a valid marker to dissect virus-infected brain tissues (Figure 8G). Brain biopsies with the highest gB RNA expression had a relatively lower If $n-\beta$ response, and zVAD treatment did inhibit CC3 (Supplemental Figure 7C). Collectively, these data suggest that prevention of cGAS-dependent cell death in microglia and other immune cells in HSV-1-infected brain tissue elevated the IFN-I response in highly infected areas, thereby leading to more efficient antiviral defense without irreversible damage to the brain (Figure 9).

\section{Discussion}

PCD is an ancient cellular response to danger and can both promote and limit infection and inflammation (37). In this work, we showed extensive apoptosis, butnotprogrammed necrosis, inHSV-1-infected brains from HSE patients and an experimental HSE mouse model. Apoptosis was induced mainly in microglia and other immune cells and was mediated by the DNA-sensing cGAS/STING pathway. This pathway was also responsible for HSV-1-induced IFN-I production in microglia. Inhibition of apoptotic caspases augmented cGASdependent antiviral activity in the brain and was also associated with prolonged and augmented IFN-I production by immune cells. Thus, the IFN-inducing cGAS/STING pathway has an in-built negative regulatory mechanism, through which activated cells undergo apoptosis as an integral part of activation. This may allow induction of sufficient amounts of IFN-I to control infection, while avoiding pathological activities of IFN-I in the brain.

DNA is a major inducer of PCD that affects both defense and disease (37). For instance, retroviral DNA can induce STING-dependent apoptosis to control retroviral replication (38), radiotherapy induces pyroptosis and tissue damage through the DNA-activated absent in melanoma 2 (AIM2) inflammasome (51), and inflammatory bowel disease is associated with both STING-driven and IFN/ TNF-dependent necroptosis (52). One key unresolved question is what determines the type of PCD induced by DNA in a given situation. Regarding viral infections with neurotropic viruses, previous studies have shown that the AIM2 inflammasome is activated in enterovirus-infected neurons, leading to pyroptosis (53). Although we did observe abundant infection of neurons in the HSV-1-infected brain of both humans and mice, we did not detect GSDMD cleavage nor MLKL phosphorylation. It is interesting that HSV-1 has evolved mechanisms to block both AIM2 and necroptosis, thereby providing a potential explanation for how HSV-1 manages to protect infected neurons from necrotic forms of cell death $(54,55)$.

Through detailed analysis of different areas of HSV-1-infected mouse brains, using laser-capture microdissection or intracranial injection, we observed that IFN-I and ISG transcription were higher in brain areas with relatively low-intermediate viral loads as compared with areas with relatively high viral loads. In contrast to this, cGAS-dependent apoptosis was most abundant in areas with a high viral load. In vitro, microglia-induced Ifnb expression and apoptosis displayed different dose dependencies, with IFN induction peaking at low-medium viral doses, whereas the apoptosis response was most prominent at high doses. These data suggest that immune cells in infected parts of the brain produce IFN-I in a cGAS-dependent manner as an immediate response to sensing viral DNA. If the viral burden increases further or, potentially, if it persists for a longer time, the cGAS/STING pathway may drive the local immune cells into apoptosis. This is in agreement with previous data suggesting that strong STING signaling favors proapoptotic responses (39). In the brain, this may represent a negative regulatory mechanism to control IFN-I expression, and hence to limit irreversible immunopathology. Indeed, several studies have described a link between the CGAS/STING pathway, PCD pathways, and negative regulation of IFN-I (49, 56-58). Collectively, this series of studies together with our present work suggests that activation of PCD pathways, in response to immunostimulatory DNA, represent a broadly used mechanism to control IFN-I responses.

The mechanistic understanding of how the STING signaling strength determines whether microglia induce IFN- $\beta$ response or undergo apoptosis remains to be clarified. At the molecular level, we found that HSV-1-induced, STING-dependent cell death in microglia and other immune cells in HSV-1-infected brains was dependent on IRF3 but independent of IFN-I signaling. Moreover, we observed no difference between $\operatorname{Irf} 3^{-/-}$and $\operatorname{Irf3} 3^{S 1 / S 1}$ mice, the latter strain lacking IRF3 transcriptional activity but able to interact with Bax and directly induce apoptosis $(46,47)$. Finally, we observed that increased Puma mRNA expression in HSV-1-infected microglia was partially dependent on cGAS. Together, these data suggest that cGAS/STING-induced expression of proapoptotic genes, including Puma, is responsible for induction of apoptosis in the HSV-1-infected brain. This mode of STING-dependent apoptosis has previously been reported in T cells (39). Regarding the role for IFN-I in the apoptotic response, another study has shown a role for IFN-I in inducing apoptosis in acute influenza infection (59). In the present study, we observed that although HSV-1-induced apoptosis was independent of IFNAR, the viral 
A
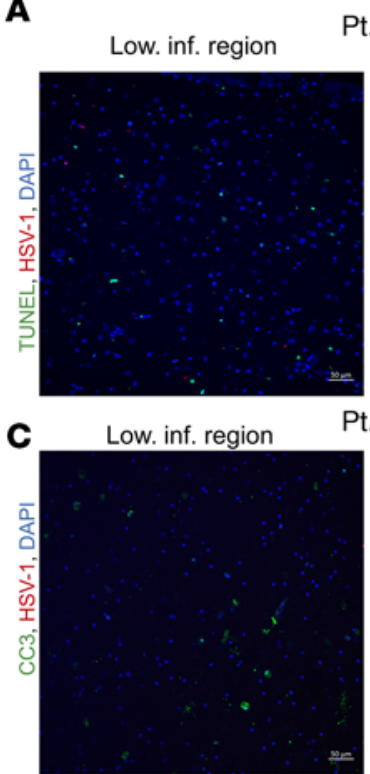

Pt. 3

Pt. 3
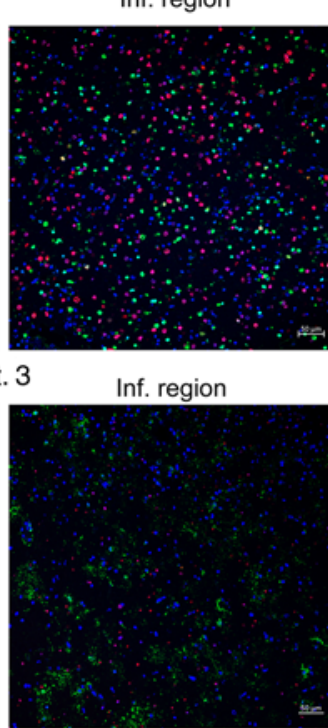

E

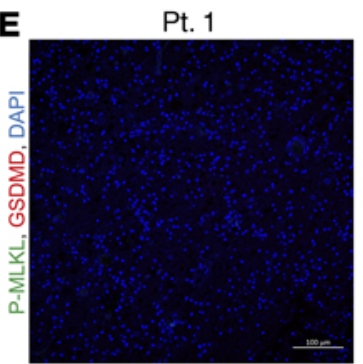

Pt. 2

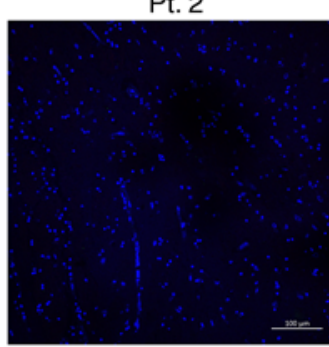

Brain trauma patient
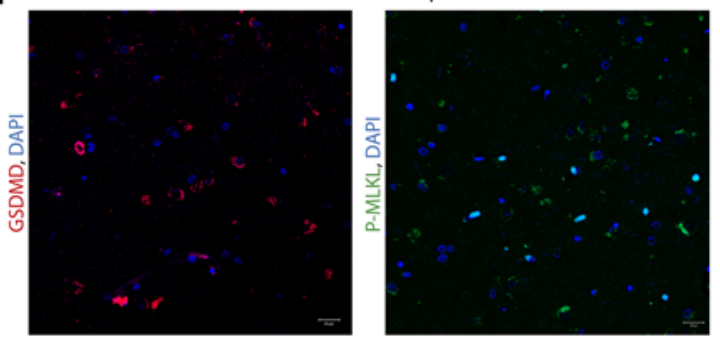

G

Pt. 2

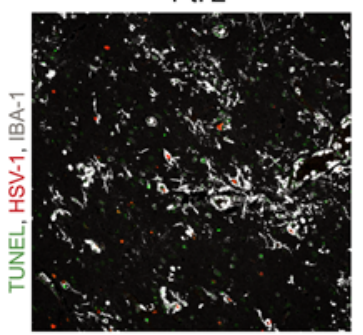

B

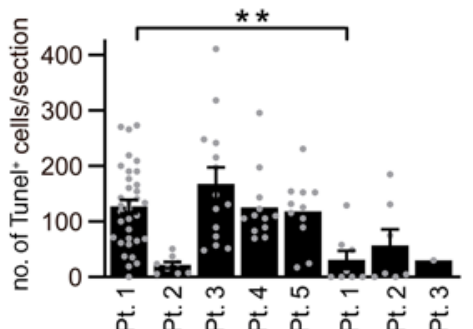

D

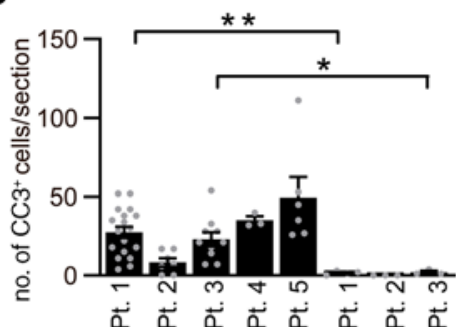

Pt. 3

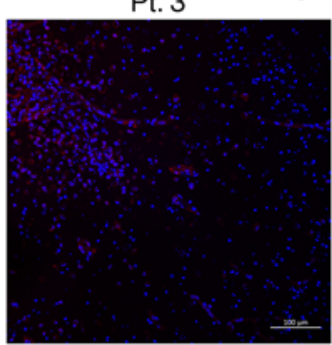

Figure 5. Apoptosis is a major form of PCD in the HSE brain. (A and D) Representative virus-infected brain sections of 5 HSE cases (Pt. 1-5) were stained for HSV-1 (ICP8, red), combined with either ( $A$ and B) TUNEL or (C and D) CC3 staining (both green). Quantification of TUNEL ${ }^{\text {pos }}$ and/or $\mathrm{CC}^{\text {pos }}$ status per section are shown as mean \pm SEM ( $n=1-34$ sections stained per patient). (E and $\mathbf{F}$ ) Representative brain sections from (E) HSE cohort and $(\mathbf{F})$ a noninfectious brain trauma patient were stained for GSDMD (red) and P-MLKL (green). Sections from the patient with brain trauma served as positive control for CCSD$M D$ and $P-M L K L$ staining. ( $\mathbf{G}$ and $\mathbf{H}$ ) Representative brain sections were stained for Iba1 (microglia; white), TUNEL (green), and HSV-1 (red). The percentages of TUNEL ${ }^{\text {pos }}$ microglia and Iba ${ }^{\text {pos }}$ cells of the total microglia population in the sections analyzed were quantified and data are shown as mean $\pm \operatorname{SEM}(n=1-5$ sections stained per patient). $P$ values were calculated by using 2-way ANOVA with Bonferroni's post hoc test (B and $\mathbf{D}$ ) and Wilcoxon rank-sum test (H). ${ }^{*} 0.01<P<0.05$; ${ }^{* *} 0.001<P<$ 0.01. Scale bars: $50 \mu \mathrm{m}(\mathbf{A}, \mathbf{C}, \mathbf{C}), 100$ $\mu \mathrm{m}(\mathbf{E})$, and $20 \mu \mathrm{m}(\mathbf{F})$.

Whereas pathological analysis of brain tissues of HSE cases indicate a role of viral cytopathic effect and local immune responses in the pathogenesis of disease, the sequence of either phenomenon and the cell types involved in the HSE disease process remain unresolved (61). Postmortem human brain tissues are rare and only reflect end-stage HSE. Experimental HSE mouse models, which recapitulate most features of HSE in humans, provide the unique opportunity to determine the virus and host factors at different times after infection, facil-

load was elevated in the Ifnar ${ }^{--}$mice. However, we cannot formally exclude that IFN-I may contribute to priming or amplification of apoptosis in the brain. HSV-1 has several strategies to inhibit IFN signaling and apoptosis (3). Although $\mathrm{CD}^{+} \mathrm{T}$ cells can induce apoptosis by secreting granzymes that induce cleavage of caspase in order to induce apoptosis of the target cells, it has also been shown that the HSV-1 latency-associated transcript can protect neurons from granzyme-induced apoptosis (60). itating detailed studies on the interaction between IFN-I and PCD on the outcome of intracerebral HSV-1 infection. Genetic data from HSE patients have demonstrated that defects in TLR3, TRIF, and TNF receptor associated factor 3, which act specifically in the TLR3 pathway, confer susceptibility to HSE (14-16). This is also observed in patients with defects in TANK binding kinase 1 or $\operatorname{IRF3}(17,18)$, which are involved downstream of TLR3 and cGAS pathways. Studies of patient-derived stem cells and transgenic mice have shown that 
A

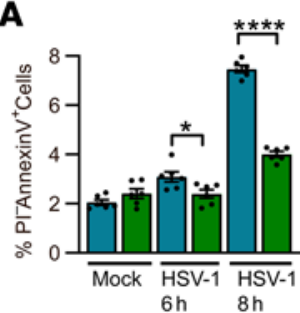

D

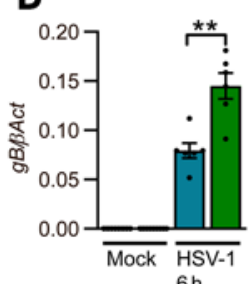

H

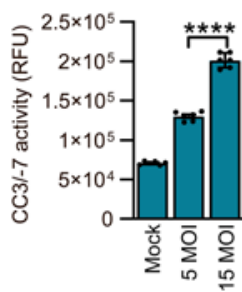

K

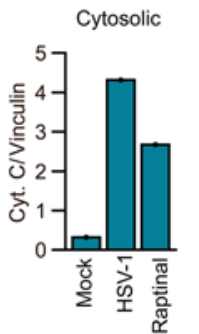

B

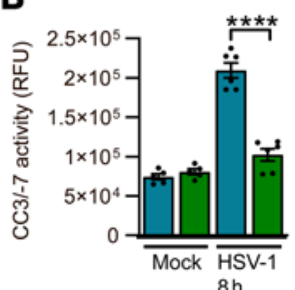

C

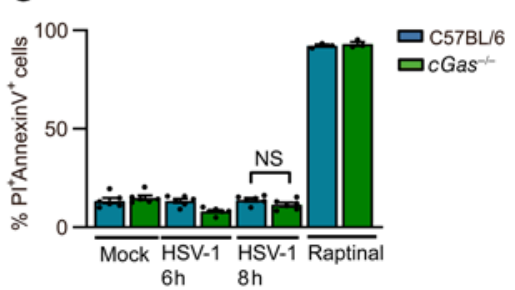

E

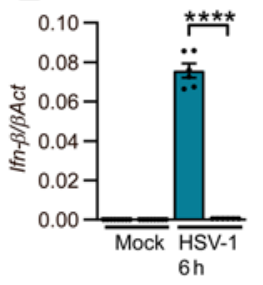

I

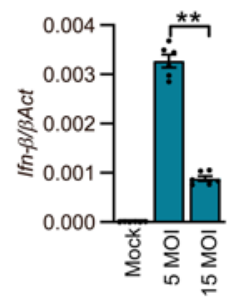

G

$\mathbf{F}$

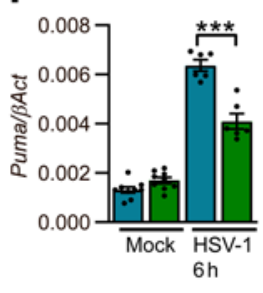

J

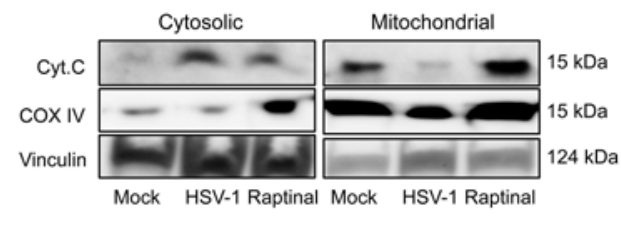

$\mathbf{L}$
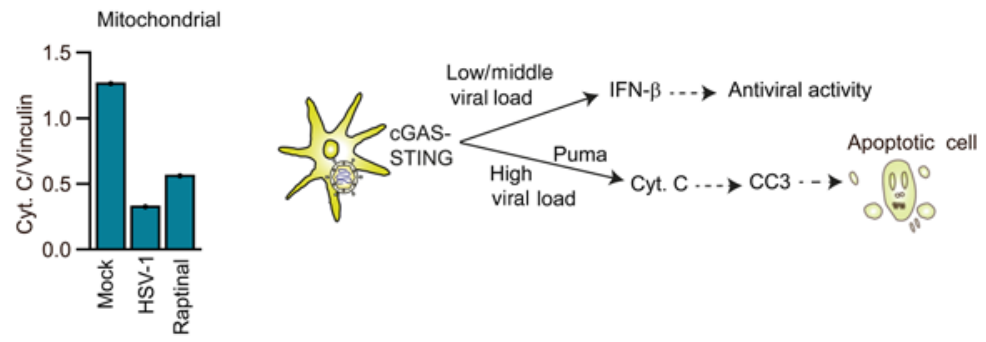

Figure 6. HSV-1 infection induces cGas/STING-dependent apoptosis in microglia. (A and C) Analysis of apoptosis in primary murine microglia after treatment with mock or HSV-1 (15 MOI) for 6 or 8 hours by flow cytometry using annexin $\mathrm{V}$ antibody and PI staining. Apoptosis inducer raptinal $(10 \mu \mathrm{M}, 45$ minutes) was used as positive control. (B) Lysates from microglia treated as in A were analyzed for caspase-3/7 activity 8 hours after infection. (D-F) Microglia were infected as in $\mathbf{A}$ for $\mathbf{6}$ hours and the expression of HSV-1 gB, Ifn $\beta$, and Puma was analyzed by real-time qPCR. Values were normalized to $\beta$-actin and presented as mean \pm SEM. (C) iPSC-derived microglia were infected with HSV-1 (MOI 5 and 15) or treated with cGAMP and analyzed for caspase-3/7 activity 12 hours later. (H and I) WT primary murine microglia were infected with 5 or $15 \mathrm{MOI}$ for 12 hours and caspase-3/7 activity or Ifn- $\beta$ mRNA levels were analyzed as in $\mathbf{B}$ and $\mathbf{E}$, respectively. ( $\mathbf{J}$ and $\mathbf{K}$ ) The microglia cell line BV2 was treated with either HSV-1 (15 MOI) for 8 hours or raptinal $(10 \mu \mathrm{M})$ for 15 minutes. The cytosolic and mitochondrial fractions were isolated and samples were subjected to immunoblotting with antibody against cytochrome c, COX IV (mitochondria marker), or vinculin. Densitometry was used to quantify the cytochrome c release relative to vinculin and shown in K. (L) Cellular model of IFN versus apoptosis response in HSV-1-infected microglia. All figures represent at least 3 independent experiments, $n=4-6$ per group (A-I) and $n=1$ (K); $P$ values were calculated by 1-way ANOVA with Tukey's multiple-comparison test ( $\mathbf{A}$ and $\mathbf{G}$ ) and 2-tailed Student's $t$ test (B-F, $\mathbf{H}$, and I). $P>0.05$ (NS, not significant); ${ }^{*} 0.01<P<$ $0.05 ;{ }^{* *} 0.001<P<0.01 ;{ }^{* *} 0.0001<P<$ $0.001 ;{ }^{* * *} P<0.0001$ the TLR3 pathway is essential for full IFN responses and subsequent control of HSV-1 infection in neurons and astrocytes $(6,7)$, whereas mouse studies have shown that cGAS is mainly involved in HSV-1 sensing and immune activation in microglia (25). These data suggest a tight interplay between these brain-resident cell types and pathways in activation of a response with maximal antiviral and minimal pathological activity. The data presented in this work showed that the cGAS/STING pathway exerted paracrine activity through the IFN-I system, but also cell-autonomous negative regulation of immune cells through activation-induced apoptosis. TLR3 has also been reported to induce apoptosis under some circumstances (62), but this seems not involved in the brain, since neurons and astrocytes did not undergo apoptosis to any significant degree during HSV-1 infection. With expanding knowledge of the signaling pathways in innate immunity, and with new technologies at the single-cell level, we can now start to understand the molecular activities that occur in tissues during infections and inflammatory diseases. This work has uncovered that immune cells present at sites of active HSV-1 replication in the brain were induced to produce IFN-I but underwent apoptosis when the immune-stimulatory signals became too strong. This mechanism may have evolved to preserve brain tissue from the damaging effect caused by long-term activation of immune cells in the brain.

\section{Methods}

Additional methods can be found in Supplemental Methods.

Mice. C57BL/6 (WT), Stingst/gt and $c \mathrm{Gas}^{-/-}$mice were bred at Taconic $\mathrm{M} \& \mathrm{~B}$ and at University of Aarhus (Denmark); Irf3 $3^{-1}, \operatorname{Irf3} 3^{s 1 / 11}$, and Ifnar ${ }^{-1-}$ mice were only bred at University of Aarhus. Ifnar/-, Stingst/gt , and $\mathrm{cGas}^{-1-}$ mice were purchased from the Jackson Laboratory. $\operatorname{Irf} 3^{-/-}$and $\operatorname{Irf} 3^{s / / s 1}$ mice were both gifts from Ganes C. Sen, Department of Immunology, Cleveland Clinic, Cleveland, Ohio, USA. Isoflurane (Abbott) or a mixture of ketamine (MSD Animal Health) and xylazine (Rompun Vet) were used to anesthetize mice. All described animal experiments have been reviewed and approved by the Danish government authorities and 
A

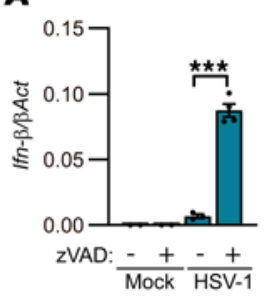

B

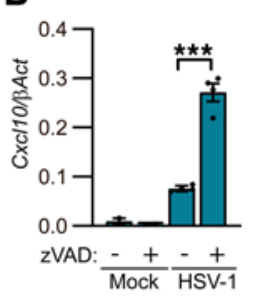

D
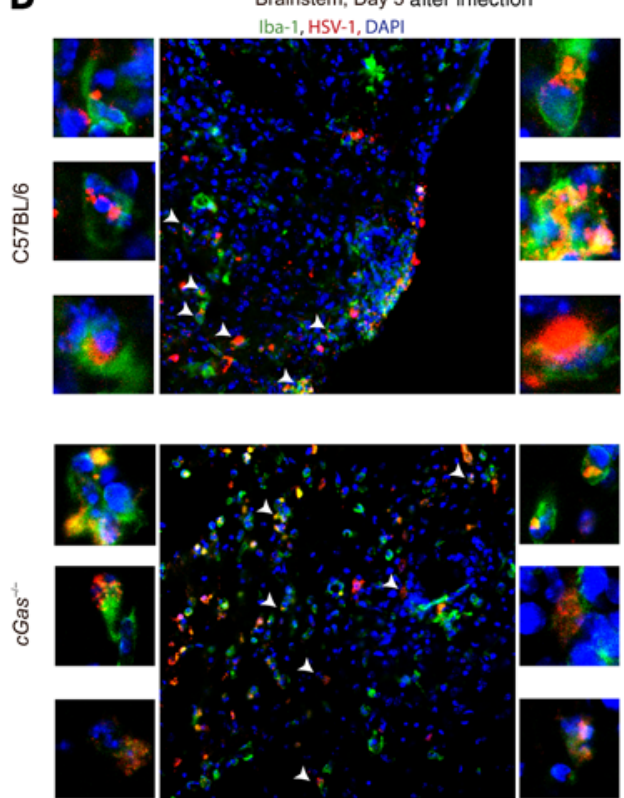

G

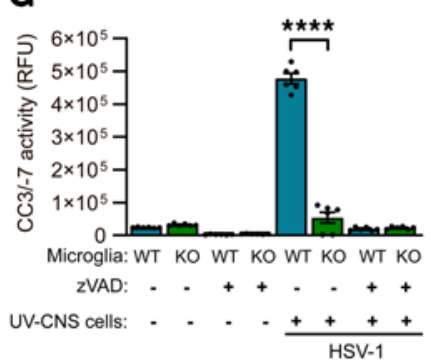

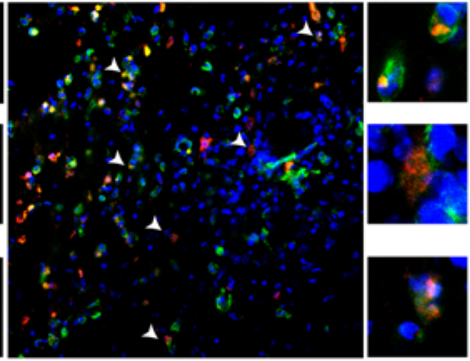

H

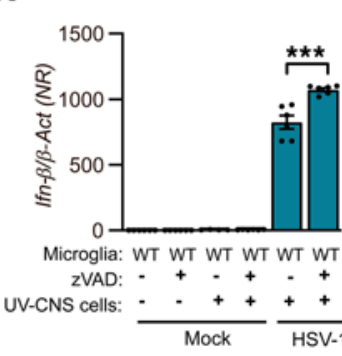

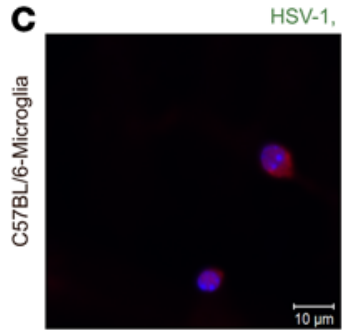

HSV-1, Iba1, DAPI

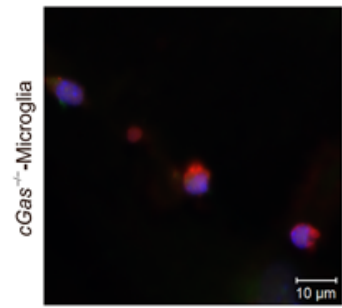

Mock CNS cells
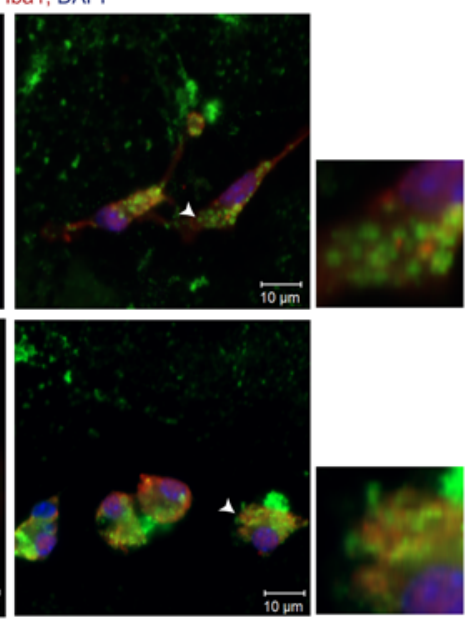

HSV-1 infected CNS cells

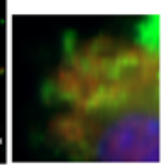

E

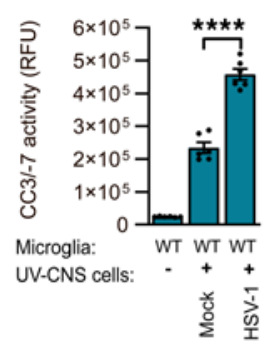

$\mathbf{F}$

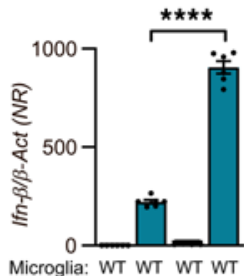

HSV-1 inf. of Microglia: :- $+\ldots$

UV-CNS cells: - -

$\frac{1}{2} \stackrel{5}{\frac{1}{1}}$

Figure 7. Microglial uptake of virus and virus-infected cells induce apoptosis to control the IFN response. (A and B) Primary murine microglia were infected with HSV-1 (15 MOI) for 12 hours in the presence of the caspase inhibitor ZVAD $(1.5 \mu \mathrm{g} / \mathrm{mL})$ or vehicle. Expression of Ifnb and Cxc/10 was analyzed by real-time qPCR and normalized to $\beta$-actin. (C) Primary murine neurons and astrocytes were mixed and infected with HSV-1-GFP (10 MOI) for 24 hours. After adding microglia for an additional 24 hours, cells were fixed and stained with DAPI (blue) and antibodies against GFP (green) and Iba-1 (red). Scale bar: $10 \mu \mathrm{m}$. Some vesicle-like structures (white arrowheads) are magnified (right). (D) Brainstems of HSV-1-infected WT and cGas ${ }^{-1-}$ mice $\left(2 \times 10^{6} \mathrm{PFU} / \mathrm{cornea}^{2}\right.$ were dissected at day 5 after infection and stained with antibodies against Iba-1 (microglia) (green), HSV-1 (polyclonal, red), and DAPI (blue). Original magnification, $\times 20$. (E) Mixed neuron and astrocyte cultures were subjected to mock or HSV-1 (10 MOI) infection for 24 hours. Virus in cells was UV inactivated and added to the microglia cultures for 12 hours. Nonphagocytosed cells were washed before caspase-3/7 activity assay of microglia was measured. (F) Microglia were HSV-1infected (15 MOI) or cultured with UV-inactivated cells as in E. After 6 hours, Ifnb expression was determined by real-time qPCR. (G and $\mathbf{H}$ ) Caspase 3/7 activity and Ifnb mRNA levels in microglia were measured after coculture with UV-inactivated neurons and astrocytes as in $\mathbf{E}$ for 12 hours in the presence of vehicle or zVAD $(1.5 \mu \mathrm{g} / \mathrm{mL})$. (I) Model of apoptosis and IFN-I response in microglia after HSV-1 infection or phagocytosis of virus-infected cells. All figures represent at least 3 independent experiments; data are presented as mean \pm SEM; $n=4-6$ per group; $P$ values were calculated by Wilcoxon rank-sum test (A and $\mathbf{B}$ ) or 2-tailed Student's $t$ test $(\mathbf{E}-\mathbf{H}){ }^{* * *} 0.0001<P<0.001 ;{ }^{* * *} P<0.0001$.

hence comply with Danish laws. All efforts were made to minimize suffering and mice were monitored daily during infection. The mice were not randomized, but after HSV-1 infection, the information about mouse strain and treatment was blinded to the investigators. No animals were excluded from the analysis. Chow and water were provided ad libitum.
Murine ocular and HSV-1 infection model. Age matched, 6-7-weekold male mice were anesthetized with i.p. injection of a mixture of ketamine (100 mg kg-1 body weight) and xylazine (10 $\mathrm{mg} \mathrm{kg}^{-1}$ body weight). We tested mice of both genders and did not find any gender differences in any of the readouts used in the current study. Both corneas 
A

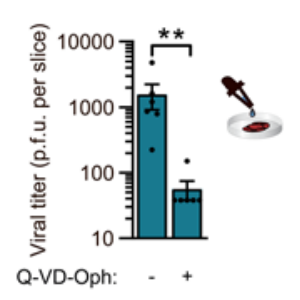

B

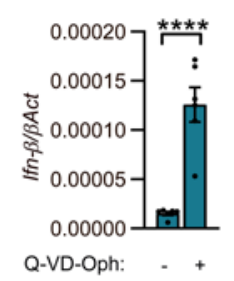

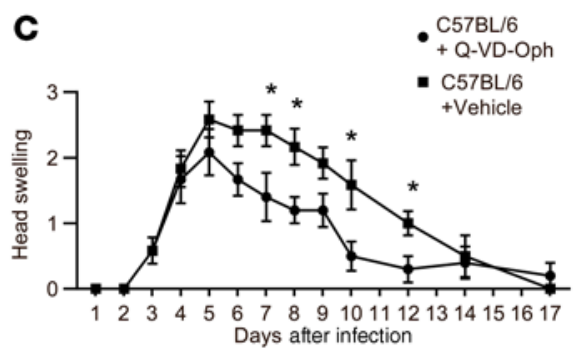

$\mathbf{E}$

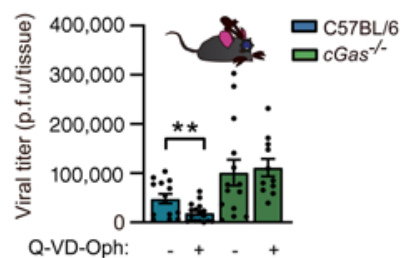

$\mathbf{F}$

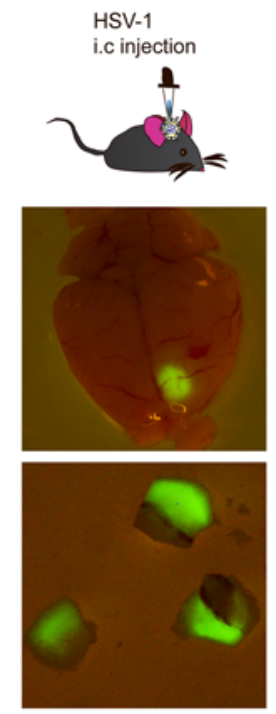

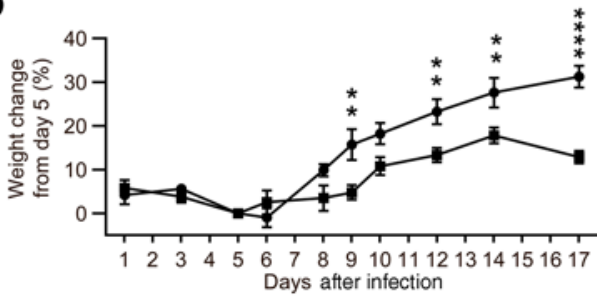

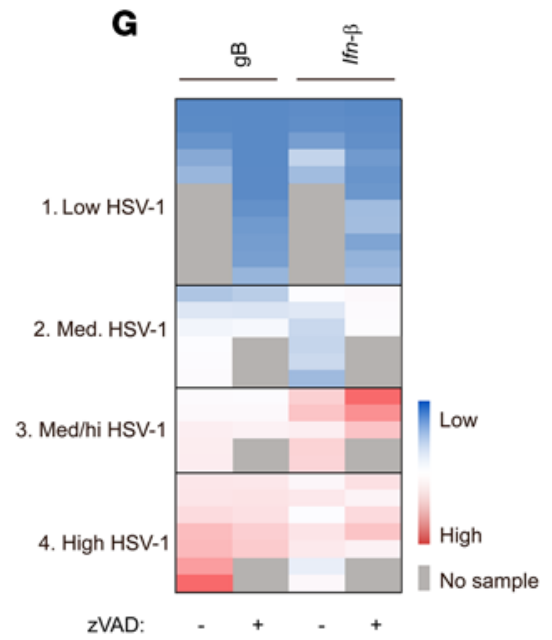

Figure 8. cGAS-dependent apoptosis limits host antiviral activity in the HSV-1-infected brain. (A and B) Organotypic brain slices from WT mice were cultured and infected with HSV-1 $\left(1 \times 10^{4} \mathrm{PFU}\right)$ for 5 days in the presence or absence of caspase inhibitor Q-VD-Oph $(100 \mu \mathrm{M})$. The viral titer and Ifnb mRNA levels in brain slices were measured and presented as mean \pm SEM. (C and $\mathbf{D})$ Mice were HSV- 1 infected $\left(4 \times 10^{7} \mathrm{PFU} / \mathrm{cornea}\right.$ ) and treated on days 5, 6, and 9 after infection with Q-VD-Oph (20 mg/kg). Head swelling and weight change were monitored. The weight was normalized to day 5 , when the treatment with Q-VD-Oph was initiated. (E) Viral titers (day 5 after infection) in the brainstems of mice infected with HSV-1 ( $2 \times 10^{6} \mathrm{PFU} / \mathrm{cornea}$ ) and then treated with vehicle or Q-VD-Oph (20 mg/kg) at days 3 and 4 after infection. (A-E) Data are presented as mean \pm SEM and represent at least 3 independent experiments, $n=6-17$ per group. $P$ values were calculated by Wilcoxon rank-sum test (A, B, and E), 2-way repeated-measures ANOVA with Sidak's multiple-comparison test $(\mathbf{C}$ and $\mathbf{D})$. ${ }^{*} 0.01<P<0.05$; ${ }^{* *} 0.001$ $<P<0.01$; ${ }^{* * *} P<0.0001$. (F and G) WT mice were infected intracranially with HSV-1-expressing GFP $\left(1 \times 10^{7} \mathrm{PFU}\right)$ and treated with the caspase inhibitor (zVAD) or vehicle as control ( $n=4-6$ mice/group) and figures represent 2 independent experiments. At 48 hours after infection, GFP-expressing brain biopsies (indicative for HSV-1 infection) were dissected. (F) Representative GFP expression in brain tissue and biopsies of HSV-1/GFP-infected mice. (C) Ifn-b and HSV-1 (gB) gene expression from the biopsies were quantified by real-time PCR. Values were normalized to $\beta$-actin and subsequently to similar biopsies from mock infected. Each row represents 1 biopsy and the biopsies are divided arbitrarily into 4 groups depending on the degree of infection defined by relative gB expression levels ( $2^{-\Delta \Delta C T}$ ): group 1: less than 3,000; group 2: 3,000-8,900; group 3: 8,900-29,000 and group 4: more than 29,000.

were scarified in a $10 \times 10$ crosshatch pattern with a 25 gauge needle and mice inoculated with HSV-1 (strain McKrae, the dosage used is indicated in the figure legends) in $5 \mu \mathrm{l}$ of infection medium (DMEM containing $200 \mathrm{IU} \mathrm{mL}^{-1}$ penicillin and $200 \mathrm{mg} \mathrm{mL}^{-1}$ streptomycin), or mock-infected with $5 \mu$ of infection medium. Mice were scored for disease and weighed at the indicated times after infection. The scoring was performed as a blinded study, with head swelling scored as 0: none, 1: minor swelling, 2: moderate swelling, 3: severe swelling.

Stereotaxic surgery and HSV-1 delivery to the mouse brain. WT 8-weekold C57BL/6 mice were anesthetized by i.p. injection of medetomidine
(40 mg/kg), midazolam (15 mg/kg), and butorphanol $(10 \mathrm{mg} / \mathrm{kg})$. Under deep anesthesia, mice were immobilized in a stereotaxic apparatus, and a hole was drilled in the skull to inject a low volume $(3 \mu \mathrm{l})$ of virus $\left(10^{7}\right.$ PFU GFP-HSV-1) in the presence of the caspase inhibitor (zVAD, 30 $\mu \mathrm{g})$ or vehicle in the brain with a 33 gauge Nanofil needle at the following mouse brain stereotaxic coordinates: $\mathrm{A} / \mathrm{P}-0.6$ to $-1.0, \mathrm{M} / \mathrm{L} 0.8$ to 1.5 , $\mathrm{D} / \mathrm{V}$-2.0. After injection, the incision was closed with 2 sutures and VetBond tissue glue (3M). At 48 hours after infection, animals were euthanized and GFP-expressing brain biopsies (indicative for HSV-1 infection) were dissected under a microscope. The biopsies were homogenized and 


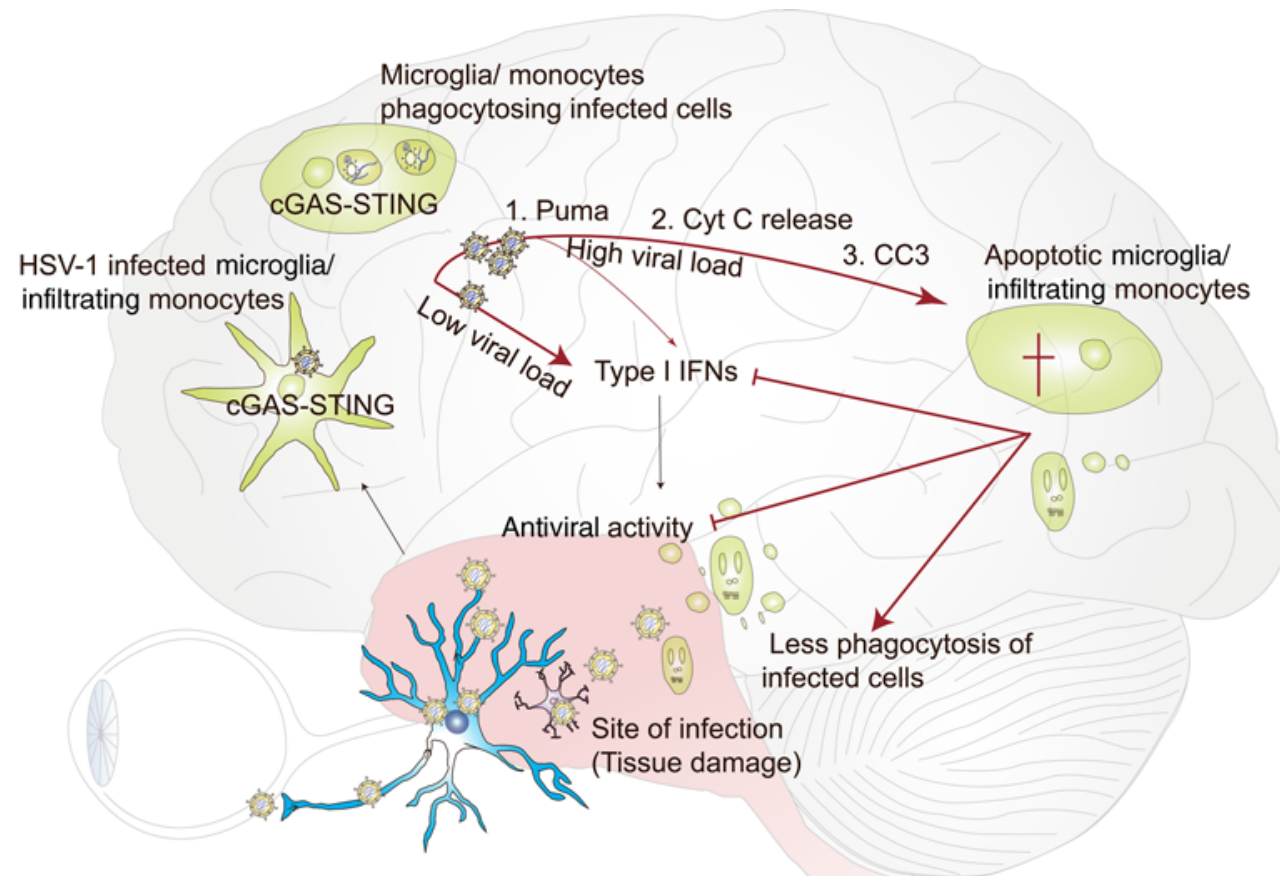

Figure 9. cGAS drives IFN-I-induced antiviral activity but also negatively regulates immune cells through apoptosis. Model illustrating how HSV- 1 infections are sensed by microglia, either by microglia being infected or upon phagocytosis of virus-infected cells. When the local viral burden is low, microglia predominantly express IFN-I, which has antiviral activity. However, when the viral burden is relatively high, cGAS/STING signaling switches to promote apoptosis by inducing Puma mRNA expression, cytochrome $c$ release from the mitochondria, and cleavage of caspase-3. This reduces the IFN-I response and potentially limits immunopathology.

divided into 2 equal parts. Total RNA was isolated from one part, and the other part was used for protein analysis.

Viruses, cells, and reagents. DMEM-F12 (Lonza) or DMEM (BioWhittaker) were supplemented with $100 \mathrm{IU} / \mathrm{mL}$ penicillin, $100 \mu \mathrm{g} /$ $\mathrm{mL}$ streptomycin, and LPS-free heat-inactivated FCS (BioWhittaker). ProLong Gold, DAPI, and recombinant mTNF- $\alpha$ were from Invitrogen. Z-VAD-FMK, nigericin, murine 3'3-cAIMP, and human 2'-3'cGAMP were from InvivoGen. LPS and PI were both obtained from Sigma-Aldrich. Both HSV-1 (McKrae) and GFP-HSV-1 (HSV-1-expressing eGFP driven by the CMV promoter) were gifts provided by David Leib, Geisel School of Medicine at Dartmouth, Lebanon, New Hampshire, USA and were grown in Vero cells. The Vero cells used were from ATCC (CCL81, RRID:CVCL_0059). The titers of the stocks used were $8 \times 10^{9}$ to $14 \times 10^{9} \mathrm{PFU} / \mathrm{mL}$, determined by standard plaque assay on Vero cells. Beriglobin was used to neutralize extracellular HSV-1 (CSL Behring) and fully neutralized the virus in the dilutions used to calculate titers. The BV-2 murine microglial cell line (RRID:CVCL_0182) was a gift provided by Anni Paulina Kleino, Aarhus Institute of Advanced Studies, Aarhus, Denmark). The BV-2 cells were cultured in DMEM including $10 \%$ FCS, $100 \mathrm{IU} / \mathrm{mL}$ penicillin, and $100 \mu \mathrm{g} / \mathrm{mL}$ streptomycin. Primary mouse neurons, microglia, and astrocytes were isolated from $c \mathrm{Gas}^{-1-}$ and WT neonatal mice as described previously (25). Spleen cells were harvested from age-matched 12-week-old female mice. Spleens were gently homogenized manually in a glass homogenizer in RPMI supplemented with $10 \%$ FCS. Erythrocytes were removed by hemolysis. The cells were passed through a $70 \mu \mathrm{m}$ pore size filter, washed, counted, and seeded 24 hours prior to stimulation.

Generation of human iPSC-derived microglia. The human iPSC line WTSIi015-A (EBiSC through Sigma-Aldrich) was maintained on Matrigel (Corning) in mTeSR1 medium (Stemcell Technologies). IPSC colonies were dissociated into single cells using TrypLE Express (Thermo Fisher Scientific) and $4 \times 10^{6}$ iPSCs seeded per Aggrewell 800 (Stemcell
Technologies) in a 24-well plate in embryonic body medium (EBM). EBM consisted of mTeSR1 medium supplemented with $10 \mu \mathrm{M}$ ROCK inhibitor, $50 \mathrm{ng} / \mathrm{mL}$ BMP-4, $20 \mathrm{ng} / \mathrm{mL} \mathrm{SCF}$, and $50 \mathrm{ng} / \mathrm{mL}$ VEGF-121 (all from Peprotech). Cells were cultured for 4 days in Aggrewells to form embryonic bodies (EBs) with half media change every day. EBs were harvested using an inverted cell strainer $(40 \mu \mathrm{m})$, and around 15 EBs were plated per 6 wells in hematopoietic medium (HM) consisting of X-VIVO 15 medium (Lonza) supplemented with $2 \mathrm{mM}$ Glutamax, $100 \mathrm{U} / \mathrm{mL}$ penicillin, $100 \mu \mathrm{g} / \mathrm{mL}$ streptomycin, $55 \mu \mathrm{M} \beta$-mercaptoethanol, $100 \mathrm{ng} / \mathrm{mL}$ human M-CSF (Peprotech), and $25 \mathrm{ng} / \mathrm{mL}$ human IL-3 (Cell Guidance Systems). Two milliliters medium was replaced every 7 days by fresh HM. After around 30 days, primitive macrophage precursors could be harvested during the media change and plated in microglia medium (MiM) into 48 wells at a density of $10^{5}$ cells $/ \mathrm{cm}^{2}$. MiM consists of contained X-VIVO 15 medium (Lonza) supplemented with 2 mM Glutamax, $100 \mathrm{U} / \mathrm{mL}$ penicillin, $100 \mu \mathrm{g} / \mathrm{mL}$ streptomycin, $55 \mu \mathrm{M}$ $\beta$-mercaptoethanol, $100 \mathrm{ng} / \mathrm{mL}$ human IL-34 (Peprotech), and $10 \mathrm{ng} /$ $\mathrm{mL}$ human GM-CSF (Peprotech). Finally, cells were differentiated in MiM for subsequent 7-10 days with full MiM change every second day.

Phagocytosis assay. Cultured mouse neurons and astrocytes were infected with mock or HSV-1 (10 MOI, McKrae), and after 1 hour, the cells were washed and replaced with fresh medium. At 24 hours after infection, cells were subjected to UV irradiation for 6 minutes to inactivate HSV-1, trypsinated, and resuspended in fresh medium. The UV inactivation of the virus was confirmed by plaque assay. The resuspended mock- and HSV-1-infected cells were added to the cultured microglia, at a 1:1 cell ratio, for phagocytosis in the presence of vehicle or zVADFMK for 3 hours. Finally, the nonphagocytosed cells were washed 3 times, replaced with fresh medium, and either the caspase-3/7 activity or mRNA expression in microglia was measured. For imaging of phagocytosis, mixed primary mouse neurons and astrocytes were seeded on coverslips and infected with mock or HSV-1-eGFP (10 MOI) for 24 hours 
before the virus was UV inactivated for 6 minutes. The cultured microglia were trypsinated, resuspended in fresh media, and added to the coverslips for 24 hours. Cells were fixed in $4 \%$ formaldehyde, permeabilized in 0.5\% Triton X-100, blocked in 5\% BSA/0.05\% Tween20/PBS, and stained overnight at $4^{\circ} \mathrm{C}$ with primary antibodies directed against GFP (1:500; rabbit polyclonal, Abcam, ) or Iba-1 (1:400; goat polyclonal, Abcam, ab5076), washed and finally stained with Alexa Fluor 488- and Alexa Fluor 647-conjugated secondary antibodies (1:500, Invitrogen, A-21441 and A-21447) for 1 hour. The cover slips were stained with DAPI, mounted, and imaged on Zeiss LSM 710.

Preparation of mouse organotypic brain slice cultures. Organotypic hippocampal slices were generated from 3-day-old postnatal pups from $\mathrm{C} 57 \mathrm{BL} / 6$ or $c \mathrm{Gas}^{-/-}$mice. For each brain, $10 \mathrm{~mL}$ of low-Na artificial cerebrospinal fluid (aCSF) was carbonated on ice with $\mathrm{CO}_{2}$ gas until some ice slashes were formed. aCSF consisted of $1 \mathrm{mM} \mathrm{CaCl}$, $10 \mathrm{mM}$ D-glucose, $4 \mathrm{mM} \mathrm{KCl}, 5 \mathrm{mM} \mathrm{MgCl}, 26 \mathrm{mM} \mathrm{NaHCO}_{3}, 234$ $\mathrm{mM}$ sucrose, and $0.1 \%$ phenol red solution. After decapitation of the pups, the extracted brain was moved gently and kept in aCSF for 1 minute before the brain and the aCSF were poured gently into a petri dish for removal of the meninges under a dissection microscope. Slices of $400 \mu \mathrm{m}$ thickness were made using a tissue chopper (Stoelting) before being moved to a warm $\left(37^{\circ} \mathrm{C}\right)$ culture medium consisting of MEM (78.8\%; Gibco), 20\% heat-inactivated horse serum (Gibco), $1 \mathrm{mM}$ L-glutamine, $1 \mathrm{mM} \mathrm{CaCl}, 2 \mathrm{mM} \mathrm{MgSO}_{4}, 170 \mathrm{nM}$ insulin, $0.0012 \%$ ascorbic acid, $12.9 \mathrm{mM}$ D-glucose, $5.2 \mathrm{mM} \mathrm{NaHCO}_{3}$, and 300 mM HEPES (all from Sigma-Aldrich) with pH 7.28 and osmolarity adjusted to 317-322. Only slices with intact brainstem regions were selected and moved to air-fluid interface-style Millicell culture inserts (30 $\mathrm{mm}$ diameter and $0.4 \mu \mathrm{m}$ pore size; Millipore) in 6-well culture plates (Thermo Fisher Scientific) with $800 \mu \mathrm{L}$ of medium added below the insert. Culture medium was changed 3 times weekly. The organotypic brain slice cultures were grown at $36^{\circ} \mathrm{C}$ in $5 \% \mathrm{CO}_{2}$ for 7 days before HSV-1 infection with $1 \times 10^{4}$ PFU of HSV1 in the presence of vehicle or Q-VD-Oph $(100 \mu \mathrm{M})$. For induction of pyroptosis, organotypic cultures were treated with LPS $(1 \mu \mathrm{g} / \mathrm{mL})$ for 4 hours and subsequently for 1 hour with nigericin $(10 \mu \mathrm{M})$. For induction of necroptosis, cultures were treated with TNF- $\alpha$ (150 ng/ $\mathrm{mL})$ and zVAD-FMK $(20 \mu \mathrm{M})$ for 20 hours, and raptinal $(100 \mu \mathrm{M}$, Sigma-Aldrich) was added for 1 hour to induce apoptosis. The individual slice cultures were cut and either fixed, stained for confocal imaging, or homogenized and used for isolation of mRNA, Western blotting, and virus plaque assay as described above.

Caspase activity. Caspase-3/7 activity of cell lysates and brainstem homogenates were performed as previously described (47). For in vitro assays, $6 \times 10^{4}$ cells were seeded in 96-well plates and infected with HSV-1 (McKrae) or treated with reagents indicated in the figure legends. Briefly, cell lysates or brainstem homogenized in RIPA buffer were used for measuring caspase-3/7 activity by the Apo-ONE Homogeneous Caspase-3/7 Assay according to protocols provided by the manufacturer (Promega). Background fluorescence levels of conditioned media for cell cultures or RIPA, in the case of brainstem homogenates, were subtracted from the fluorescence values measured in the experimental samples.

Cytochrome $\mathrm{c}$ release assay. For the cytochrome c assay, $5 \times 10^{7}$ BV-2 cells were mock- or HSV-1-infected (15 MOI; McKrae) for 8 hours or treated with raptinal $(10 \mu \mathrm{M})$ for 15 minutes. The mitochondrial and cytosolic fractions of stimulated cells were separated using the Cytochrome C Release Assay Kit (Abcam, ab65311) according to the manufacturer's protocol, and $30 \mu \mathrm{g}$ of the cytosolic and mitochondrial fractions was subjected to Western blotting.

IHC on mouse brain tissues and cells. IHC was done as previously described (25). Briefly, mice were perfused and dissected brains fixed with $4 \%$ formaldehyde, embedded in paraffin, and sections cut at 7 $\mu \mathrm{m}$ thickness. Before staining, sections were deparaffinized and rehydrolyzed, and antigens were retrieved for 30 minutes at $80^{\circ} \mathrm{C}$ using the Target Retrieval Solution (Darko). When using cells on coverslips, the cells were fixed for 10 minutes with $4 \%$ formaldehyde and permeabilized in $0.2 \%$ Triton $\mathrm{X}-100$ for 5 minutes. The samples were blocked with $1 \%$ BSA in TBS with $0.3 \%$ Triton X-100 (TBS-TX) for 1 hour at room temperature (RT). Samples were stained by incubation overnight at $4^{\circ} \mathrm{C}$ with the following primary antibodies: mouse monoclonal anti-HSV-1 VP5 (1:300; Abcam, clone 3B6), rabbit polyclonal anti-HSV-1 (1:500; Dako Cytomation, B0116), rabbit polyclonal anticleaved caspase-3 (1:300; Cell Signaling Technology, Asp175), goat polyclonal anti-Iba1(1:400; Abcam, ab5076), mouse monoclonal antiNeuN (1:300; Millipore, clone A60), mouse monoclonal anti-GFAP (1:400; Sigma-Aldrich, clone G-A-5), rabbit monoclonal anti-GSDMD (Abcam, EPR19828), rabbit polyclonal anti-Phospho-RIPK3 (Cell Signaling Technology, Thr231/Ser232), rabbit polyclonal anti-MLKL (Abcam, ab172868), or mouse anti-cytochrome c (1:200; Abcam, ab65311). As control for staining, we used secondary antibody alone or isotype control if the primary antibody was monoclonal. For identification of $\mathrm{PI}^{\text {pos }}$ cells in the brain tissues, PI $(200 \mu \mathrm{L}$ of $1 \mu \mathrm{g} / \mu \mathrm{L}$ PI solution) was i.p. administered to mice at 90 minutes before perfusion and fixation of the mice. TUNEL staining was done on brain sections using the Apoptag S7111 kit (Millipore) according to the manufacturer's instructions. After several washes in TBS-TX, the appropriate secondary antibodies coupled to Alexa Fluor 647, 568, or 488 (1:500; Invitrogen) were incubated for 1 hour at RT. Nuclei were stained with DAPI for 6 minutes. Finally, the sections were washed 5 times with TBS-TX and mounted with ProLong Gold. Sections were imaged on a Zeiss LSM 710 or LSM 800 laser scanning microscope or Leica Leitz DMRB fluorescence microscope. Zen 2012 acquisition software and Image $(\mathrm{NIH})$ were used for imaging and analysis.

IHC on human brain tissue. FFPE brain specimens of 5 deceased patients with HSE and 1 control patient who died of severe head trauma were obtained postmortem for diagnostic purposes. According to the institutional "opt-out" system (Erasmus Medical Centre), which is defined by the National Code of Good Conduct (Dutch: Code Goed Gebruik, May 2011), these surplus human brain tissues were available for the current study. Sections of $4 \mu \mathrm{m}$ thickness were cut from the human FFPE brain biopsies and cooked at $60^{\circ} \mathrm{C}$ for 1 hour prior to being deparaffinized. Antigen retrieval was performed with citric acid for 15 minutes and TrueBlack Lipofuscin Autofluorescence Quencher (Biotium) was applied to limit background fluorescence. TUNEL staining was done on brain sections using the Apoptag S7111 kit (Millipore) according to the manufacturer's instructions. Subsequently, sections were treated with $3 \% \mathrm{H}_{2} \mathrm{O}_{2}$ and $1 \%$ absolute methanol for 30 minutes and blocked with $0.1 \%$ BSA diluted in TBS before staining with the following primary antibodies: polyclonal rabbit anti-GFAP (1:10000; Dako, Z0334), polyclonal rabbit anti-Iba1 (1:500; Wako, 019-19741), rabbit monoclonal MLKL (phospho S358; 1:250; Abcam, EPR9514), mouse monoclonal anti-GSDMD (1:1000; Abnova, 3F12-1B2), polyclonal rabbit anti-cleaved caspase-3 (1:50; Promega, G7481), monoclonal mouse anti-HSV-1 (1:50; Cell 
Marque, 10A3), or monoclonal mouse anti-CD45 (1:50; Dako, $2 \mathrm{~B} 11+$ $\mathrm{PD} 7 / 26)$. After overnight incubation with the primary antibodies in TBSTX with 0.1\% BSA, sections were washed extensively with TBS-TX and subsequently incubated for 1 hour at RT with the appropriate secondary antibodies: Alexa Fluor 647 goat anti-rabbit (1:250; Invitrogen, A21244) or Alexa Fluor 555 goat anti-mouse (1:250; Invitrogen, A21127). Finally, sections were incubated with Hoechst dye (1:1000; 20 mM 33342 solution, Invitrogen) for 10 minutes at RT to visualize nuclei and mounted with ProLong Diamond Antifade Mountant for imaging using a Zeiss LSM 700 confocal microscope as described previously (63).

RNA isolation, real-time qPCR, multiplex $q P C R$ on Biomarker (Fluidigm). Tissues were homogenized with steel beads (Qiagen) in a Tissuelyser (II) (Qiagen) in PBS and immediately used for RNA isolation. RNA from brainstem or cell cultures was isolated using the High Pure RNA Isolation Kit (Roche) and real-time PCR was done as described (25). mRNA levels of interest were normalized to the mouse housekeeping gene $\beta$-actin using the formula $2^{\mathrm{Ct}(\beta \text {-actin }}$ $\mathrm{mRNA})$-Ct(target $\mathrm{mRNA}$. The mRNA from brainstem or laser-guided dissected tissue fragments was processed to cDNA, and specific targets were preamplified prior to multiplex qPCR on the BioMarker (Fluidigm) as described (25), including using the primers Ifit1 (Mm00515153_m1), Mx2 (Mm00488995_m1), b-Act (Mm00607939_s1), and TNF-a (Mm00443260_g1) from Applied Biosystems.

Isolation of brainstem using laser-capture microdissection. The mice were perfused with PBS and dissected brains embedded with Tissue-Tek OCT compound (Sakura) in a plastic base mold and snap-frozen in a cell culture plate floating on liquid nitrogen. Cross-sections of the brain tissue were sectioned at $7 \mu \mathrm{m}$ thickness on a cryostat and mounted onto Arcturus PEN membrane glass slides (Life Technologies) and frozen at $-80^{\circ} \mathrm{C}$. Adjacent $7 \mu \mathrm{m}$ sections were postfixed in $4 \%$ formaldehyde and stained with antibody rabbit polyclonal anti-HSV-1 (1:500; DakoCytomation, B0116) followed by $\mathrm{H} \& \mathrm{E}$ staining for histological orientation as previously described (25). The process of making tissue sections was performed as quickly as possible ( $\sim 2-3$ minutes) to prevent thawing of the cut sections, and extreme caution was taken to ensure RNase-free performance. The PEN membranes were stained with Histogene LCM Frozen Section Staining Kit (Life Technologies) using the manufacturer's protocol. The different degrees of infected areas $\left(50 \mu \mathrm{m}^{2}\right)$ were cut with Veritas Microdissection Instrument (Arcturus Bioscience) with a laser spot size $2 \mu \mathrm{m}$, pulse power $70 \mathrm{~mW}$, and pulse duration $2000 \mu \mathrm{s}$. RNA from the laser-captured brainstem sections was then extracted using Pico Pure RNA Isolation Kit (Applied Biosystems) and realtime qPCR was performed as described above.

Flow cytometry. Flow cytometry was performed as previously described (25). Briefly, the brainstems from adult mouse brains were made into single-cell suspension by carefully triturating with Pasteur glass pipettes (VWR) with decreasing diameter until it became a homogenous cell suspension. Large debris and cell clusters were removed by filtration through a $70 \mu \mathrm{m}$ cell strainer (BD Biosciences) and myelin debris was reduced by a 3-density step Percoll gradient (Sigma-Aldrich). Subsequently, cells were blocked, washed, and stained with rabbit polyclonal anti-cleaved caspase-3 (1:500; Cell Signaling Technology, Asp175), followed by staining with secondary antibody, goat anti-rabbit Alexa Fluor 488 (1:500; Invitrogen, A-11008). Finally, the samples were fixed in $1 \%$ formaldehyde and analyzed by NovoCyte (ACEA Biosciences). Annexin V and PI staining of primary microglia was done using Alexa Fluor 488 Annexin V/Dead cell Apoptosis kit (Invitrogen, V13241) according to the manufacturer's protocol.

MRI. All animals were scanned using a $9.4 \mathrm{~T}$ preclinical MRI system (Bruker Biospin) with a BGA-12HP gradient set. Imaging was performed using a cross-coil setup with a $76 \mathrm{~mm}$ quadrature coil for excitation and a bore-mounted 4-element cryosurface coil for signal reception (Bruker Biospin). Prior to scanning, animals were anesthetized with $4 \%-5 \%$ isoflurane and transferred to the scanner bed where anesthesia was maintained with a $1.5 \%-2.5 \%$ isoflurane air/oxygen mixture as described previously $(64,65)$. Animal body temperature was monitored and kept stable $\left(36.4^{\circ} \mathrm{C} \pm 0.7^{\circ} \mathrm{C}\right)$ throughout using a temperature-controlled circulating warm water blanket placed over the animal during scans. Similarly, animal respiration rate $(131 \pm 37$ breaths/minute) was monitored throughout the MRI session using a physiological monitoring system. Small adjustments to isoflurane flow were performed during scanning to keep temperature and respiration rate normal and stable. Reported values are average \pm 1 SD over all animals. Anatomical images were acquired using a rapid acquisition with relaxation enhancement (RARE) sequence. Axial images were acquired with TR/effective TE of 2200/22 ms, $364 \times 364$ matrix, 28 slices, slice thickness of $0.5 \mathrm{~mm}$, in-plane resolution of $0.05 \mathrm{~mm}$, RARE factor of 4 , and averages of 4 . The total time for an in vivo anatomical scan was 13.3 minutes. Data were subsequently transferred to MATLAB (MathWorks) for processing and analysis.

Statistics. For statistical analysis of data, we used 2-tailed Student's $t$ test when the data exhibited normal distribution, and Wilcoxon rank-sum test when the data set did not pass the normal distribution test. When comparing more than 2 groups, multiple-comparison 1-way ANOVA was used with Tukey's or Kruskal-Wallis multiple-comparison test or 2-way ANOVA with either Bonferroni's or Sidak's multiple-comparison test was used, as indicated in figure legends. For survival studies, the $P$ values were calculated using log-rank (Mantel-Cox) test. $P$ values equal to 0.05 were considered to reflect statistically significant differences between compared groups. All experimental data were reliably reproduced in 2 or more individual biological replicates. GraphPad Prism 8 software was used for statistical analyses. No measurement was excluded for statistical analysis.

Study approval. All experiments involving animals were approved in advance by the Animal Ethics Committee at the Danish Veterinary and Food Administration (Stationsparken 31-33, 2600 Glostrup, Denmark) and were carried out in accordance with the Danish Animal Welfare Act for the Care and Use of Animals for Scientific Purposes. The work involving surplus human brain material, obtained from authorized Dutch Biobanks, was approved by the medical ethical committees of the Erasmus Medical Centre and VU Medical Center (Amsterdam, The Netherlands) for the respective biobanks. The study was performed in accordance with the Declaration of Helsinki, and specimens were obtained and used in accordance with national regulations.

\section{Author contributions}

LSR, GMGMV, and SRP designed the research. LSR, ASR, DNT, GKP, $\mathrm{AKH}, \mathrm{MG}, \mathrm{SF}, \mathrm{CB}, \mathrm{MKT}$, and ARK developed methodologies and performed the research. LSR, ASR, DNT, GKP, AKH, MG, SF, CB, MKT, ARK, GMGMV, and SRP analyzed data. LSR, ASR, DNT, AKH, MG, MHV, ARK, and BH visualized data. MHV, BH, MHC, JRN, GCS, and GMGMV provided reagents. LSR, PB, LA, HZ, GMGMV, and SRP 
supervised. LSR, PB, LA, THM, JRN, HZ, GMGMV, and SRP reviewed and edited the manuscript. LSR and SRP wrote the original manuscript.

\section{Acknowledgments}

This work was funded by the European Research Council (ERC-AdG ENVISION; 786602); the Novo Nordisk Foundation (NNF18OC0030274), the Lundbeck Foundation (R198-2015171; R268-2016-3927), and the Swedish Council (\#2018-02463). $\mathrm{HZ}$ is a Wallenberg Scholar supported by grants from the Swedish Research Council (\#2018-02532), the European Research Council (\#681712), Swedish State Support for Clinical Research
(\#ALFGBG-720931), and the UK Dementia Research Institute at UCL. The technical assistance of Kirsten Stadel Petersen, T. Khemai-Mehraban, Sadegh Nabavi, Anne-Katrine Vestergaard, Mahboobeh Amoushahi, Darshana Dattatraya Kadekar, Helle Hasager Damkier, Benedicte Parm Ulhøi, and Mathilde B.H. Thomsen is greatly appreciated. We also acknowledge AU Health Bioimaging Core Facility and AU FACS Core facility for the use of the facilities.

Address correspondence to: Søren Riis Paludan, Department of Biomedicine, Aarhus University, Høegh-Guldbergsgade 10, DK-8000 Aarhus C, Denmark. Phone: 4528992066; Email: srp@biomed.au.dk.
1. Tyler KL. Acute viral encephalitis. N EnglJ Med. 2018;379(6):557-566.

2. Roos KL. Encephalitis. Handb Clin Neurol. 2014;121:1377-1381.

3. Paludan SR, Bowie AG, Horan KA, Fitzgerald KA. Recognition of herpesviruses by the innate immune system. Nat Rev Immunol. 2011;11(2):143-154.

4. Ouwendijk WJ, Laing KJ, Verjans GM, Koelle DM. T-cell immunity to human alphaherpesviruses. Curr Opin Virol. 2013;3(4):452-460.

5. Whitley RJ. Herpes simplex viruses. In: Knipe DM, Howley PM eds. Fields Virology. Lippincott, Williams \& Wilkins; 2001:2461-2509.

6. Reinert LS, et al. TLR3 deficiency renders astrocytes permissive to herpes simplex virus infection and facilitates establishment of CNS infection in mice. JClin Invest. 2012;122(4):1368-1376.

7. Lafaille FG, et al. Impaired intrinsic immunity to HSV-1 in human iPSC-derived TLR3-deficient CNS cells. Nature. 2012;491(7426):769-773.

8. Michalopoulos GK, DeFrances MC. Liver regeneration. Science. 1997;276(5309):60-66.

9. Takeo M, Lee W, Ito M. Wound healing and skin regeneration. Cold Spring Harb Perspect Med. 2015;5(1):a023267.

10. Eimer WA, et al. Alzheimer's disease-associated $\beta$-amyloid is rapidly seeded by herpesviridae to protect against brain infection. Neuron. 2018;99(1):56-63.e3.

11. Orvedahl A, MacPherson S, Sumpter R, Tallóczy Z, Zou Z, Levine B. Autophagy protects against Sindbis virus infection of the central nervous system. Cell Host Microbe. 2010;7(2):115-127.

12. Zhang SY, et al. Inborn errors of RNA lariat metabolism in humans with brainstem viral infection. Cell. 2018;172(5):952-965.e18.

13. Lafaille FG, et al. Human SNORA31 variations impair cortical neuron-intrinsic immunity to HSV-1 and underlie herpes simplex encephalitis. Nat Med. 2019;25(12):1873-1884.

14. Zhang SY, et al. TLR3 deficiency in patients with herpes simplex encephalitis. Science. 2007;317(5844):1522-1527.

15. Pérez de Diego R, et al. Human TRAF3 adaptor molecule deficiency leads to impaired Toll-like receptor 3 response and susceptibility to herpes simplex encephalitis. Immunity. 2010;33(3):400-411.

16. Sancho-Shimizu V, et al. Herpes simplex encephalitis in children with autosomal recessive and dominant TRIF deficiency. JClin Invest. 2011;121(12):4889-4902.

17. Herman M, et al. Heterozygous TBK1 mutations impair TLR3 immunity and underlie herpes simplex encephalitis of childhood. J Exp Med. 2012;209(9):1567-1582.

18. Andersen LL, et al. Functional IRF3 deficiency in a patient with herpes simplex encephalitis. JExp Med. 2015;212(9):1371-1379.

19. Alexopoulou L, Holt AC, Medzhitov R, Flavell RA. Recognition of double-stranded RNA and activation of NF-kappaB by Toll-like receptor 3 . Nature. 2001;413(6857):732-738.

20. Yamamoto M, et al. Cutting edge: a novel Toll/ IL-1 receptor domain-containing adapter that preferentially activates the IFN-beta promoter in the Toll-like receptor signaling. J Immunol. 2002;169(12):6668-6672.

21. Yoneyama M, et al. The RNA helicase RIG-I has an essential function in double-stranded RNA-induced innate antiviral responses. Nat Immunol. 2004;5(7):730-737.

22. Seth RB, Sun L, Ea CK, Chen ZJ. Identification and characterization of MAVS, a mitochondrial antiviral signaling protein that activates NF-kappaB and IRF 3. Cell. 2005;122(5):669-682.

23. Sun L, Wu J, Du F, Chen X, Chen ZJ. Cyclic GMPAMP synthase is a cytosolic DNA sensor that activates the type I interferon pathway. Science. 2013;339(6121):786-791.

24. Ishikawa H, Barber GN. STING is an endoplasmic reticulum adaptor that facilitates innate immune signalling. Nature. 2008;455(7213):674-678.

25. Reinert LS, et al. Sensing of HSV-1 by the cGASSTING pathway in microglia orchestrates antiviral defence in the CNS. Nat Commun. 2016;7:13348.

26. Li XD, Wu J, Gao D, Wang H, Sun L, Chen ZJ. Pivotal roles of cGAS-cGAMP signaling in antiviral defense and immune adjuvant effects. Science. 2013;341(6152):1390-1394.

27. Ishikawa H, Ma Z, Barber GN. STING regulates intracellular DNA-mediated, type I interferon-dependent innate immunity. Nature. 2009;461(7265):788-792.

28. Rodero MP, Crow YJ. Type I interferon-mediated monogenic autoinflammation: the type I interferonopathies, a conceptual overview. JExp Med. 2016;213(12):2527-2538.

29. Crow YJ, Manel N. Aicardi-Goutières syndrome and the type I interferonopathies. Nat Rev Immunol. 2015;15(7):429-440.

30. Prinz M, Jung S, Priller J. Microglia biology: one century of evolving concepts. Cell. 2019;179(2):292-311.

31. Seitz S, Clarke P, Tyler KL. Pharmacologic depletion of microglia increases viral load in the brain and enhances mortality in murine models of flavivirus-induced encephalitis. J Virol. 2018;92(16):e00525-18.

32. Wheeler DL, Sariol A, Meyerholz DK, Perlman S. Microglia are required for protection against lethal coronavirus encephalitis in mice. JClin Invest. 2018;128(3):931-943.

33. Sierra A, et al. Microglia shape adult hippocampal neurogenesis through apoptosis-coupled phagocytosis. Cell Stem Cell. 2010;7(4):483-495.

34. Parkhurst CN, et al. Microglia promote learning-dependent synapse formation through brain-derived neurotrophic factor. Cell. 2013;155(7):1596-1609.

35. Nissen JC, Thompson KK, West BL, Tsirka SE. Csf1R inhibition attenuates experimental autoimmune encephalomyelitis and promotes recovery. Exp Neurol. 2018;307:24-36.

36. Shi Y, et al. Microglia drive APOE-dependent neurodegeneration in a tauopathy mouse model. JExp Med. 2019;216(11):2546-2561.

37. Paludan SR, Reinert LS, Hornung V. DNA-stimulated cell death: implications for host defence, inflammatory diseases and cancer. Nat Rev Immunol. 2019;19(3):141-153.

38. Sze A, Belgnaoui SM, Olagnier D, Lin R, Hiscott $\mathrm{J}$, van Grevenynghe J. Host restriction factor SAMHD1 limits human $\mathrm{T}$ cell leukemia virus type 1 infection of monocytes via STING-mediated apoptosis. Cell Host Microbe. 2013;14(4):422-434.

39. Gulen MF, et al. Signalling strength determines proapoptotic functions of STING. Nat Commun. 2017;8(1):427.

40. Gaidt MM, et al. The DNA inflammasome in human myeloid cells is initiated by a STINGCell death program upstream of NLRP3. Cell. 2017;171(5):1110-1124.e18.

41. Schock SN, et al. Induction of necroptotic cell death by viral activation of the RIG-I or STING pathway. Cell Death Differ. 2017;24(4):615-625.

42. Stacey KJ, Ross IL, Hume DA. Electroporation and DNA-dependent cell death in murine macrophages. Immunol Cell Biol. 1993;71 ( Pt 2):75-85.

43. Nassour J, et al. Autophagic cell death restricts chromosomal instability during replicative crisis. Nature. 2019;565(7741):659-663.

44. Garrity MM, Burgart LJ, Riehle DL, Hill EM, Sebo TJ, Witzig T. Identifying and quantifying apoptosis: navigating technical pitfalls. Mod Pathol. 2003;16(4):389-394.

45. Paludan SR. Activation and regulation of DNA-driven immune responses. Microbiol Mol 
Biol Rev. 2015;79(2):225-241.

46. Chattopadhyay S, Yamashita M, Zhang Y, Sen GC. The IRF-3/Bax-mediated apoptotic pathway, activated by viral cytoplasmic RNA and DNA, inhibits virus replication. J Virol. 2011;85(8):3708-3716.

47. Chattopadhyay S, Kuzmanovic T, Zhang Y, Wetzel JL, Sen GC. Ubiquitination of the transcription factor IRF-3 activates RIPA, the apoptotic pathway that protects mice from viral pathogenesis. Immunity. 2016;44(5):1151-1161.

48. Garrido C, Galluzzi L, Brunet M, Puig PE, Didelot C, Kroemer G. Mechanisms of cytochrome c release from mitochondria. Cell Death Differ. 2006;13(9):1423-1433.

49. Ning X, et al. Apoptotic caspases suppress type I interferon production via the cleavage of cGAS, MAVS, and IRF3. Mol Cell. 2019;74(1):19-31.e7.

50. Braun JS, Prass K, Dirnagl U, Meisel A, Meisel C. Protection from brain damage and bacterial infection in murine stroke by the novel caspase-inhibitor Q-VD-OPH. Exp Neurol. 2007;206(2):183-191.

51. Hu B, et al. The DNA-sensing AIM2 inflammasome controls radiation-induced cell death and tissue injury. Science. 2016;354(6313):765-768.
52. Aden K, et al. ATG16L1 orchestrates interleukin-22 signaling in the intestinal epithelium via cGASSTING. J Exp Med. 2018;215(11):2868-2886.

53. Yogarajah T, Ong KC, Perera D, Wong KT. AIM2 inflammasome-mediated pyroptosis in enterovirus A71-infected neuronal cells restricts viral replication. Sci Rep. 2017;7(1):5845.

54. Maruzuru Y, et al. Herpes simplex virus 1 VP22 inhibits AIM2-dependent inflammasome activation to enable efficient viral replication. Cell Host Microbe. 2018;23(2):254-265.e7.

55. Guo H, et al. Herpes simplex virus suppresses necroptosis in human cells. Cell Host Microbe. 2015;17(2):243-251.

56. Rongvaux A, et al. Apoptotic caspases prevent the induction of type I interferons by mitochondrial DNA. Cell. 2014;159(7):1563-1577.

57. White MJ, et al. Apoptotic caspases suppress mtDNA-induced STING-mediated type I IFN production. Cell. 2014;159(7):1549-1562.

58. Corrales L, Woo SR, Williams JB, McWhirter SM, Dubensky TW, Gajewski TF. Antagonism of the STING pathway via activation of the AIM2 inflammasome by intracellular DNA. J Immunol. 2016;196(7):3191-3198.

59. Davidson S, Crotta S, McCabe TM, Wack A.
Pathogenic potential of interferon $\alpha \beta$ in acute influenza infection. Nat Commun. 2014;5:3864.

60. Jiang $X$, et al. The herpes simplex virus type 1 latency-associated transcript can protect neuron-derived C1300 and Neuro2A cells from granzyme B-induced apoptosis and CD8 T-cell killing. JVirol. 2011;85(5):2325-2332.

61. Mancini M, Vidal SM. Insights into the pathogenesis of herpes simplex encephalitis from mouse models. Mamm Genome. 2018;29(7-8):425-445.

62. Salaun B, Coste I, Rissoan MC, Lebecque SJ, Renno T. TLR3 can directly trigger apoptosis in human cancer cells. J Immunol. 2006;176(8):4894-4901.

63. Ouwendijk WJ, et al. T-cell tropism of simian varicella virus during primary infection. $P L O S$ Pathog. 2013;9(5):e1003368.

64. Khan AR, Hansen B, Wiborg O, Kroenke CD, Jespersen SN. Diffusion MRI and MR spectroscopy reveal microstructural and metabolic brain alterations in chronic mild stress exposed rats: a CMS recovery study. Neuroimage. 2018;167:342-353.

65. Hansen B, et al. White matter biomarkers from fast protocols using axially symmetric diffusion kurtosis imaging. NMR Biomed. 2017;30(9). 